\title{
Overexpression of SIGRAS7 Affects Multiple Behaviors Leading to Confer Abiotic Stresses Tolerance and Impacts Gibberellin and Auxin Signaling in Tomato
}

\author{
Sidra Habib, Muhammad Waseem $(\mathbb{D}$, Ning Li, Lu Yang, and Zhengguo Li $\mathbb{D}$ \\ School of Life Science, Chongqing University, Chongqing 400044, China \\ Correspondence should be addressed to Zhengguo Li; 11400065@cqu.edu.cn
}

Received 8 September 2018; Revised 8 January 2019; Accepted 27 January 2019; Published 1 July 2019

Academic Editor: Wilfred van IJcken

Copyright (C) 2019 Sidra Habib et al. This is an open access article distributed under the Creative Commons Attribution License, which permits unrestricted use, distribution, and reproduction in any medium, provided the original work is properly cited.

\begin{abstract}
Abiotic stresses remain the key environmental issues that reduce plant development and therefore affect crop production. Transcription factors, such as the GRAS family, are involved in various functions of abiotic stresses and plant growth. The GRAS family of tomato (Solanum lycopersicum), SlGRAS7, is described in this study. We produced overexpressing SlGARS7 plants to learn more about the GRAS transcription factors. Plants overexpressing SlGARS7 (SlGRAS7-OE) showed multiple phenotypes related to many behaviors, including plant height, root and shoot length, and flowering time. We observed that many genes in the SlGRAS7-OE seedlings that are associated with auxin and gibberellin (GA) are downregulated and have altered sensitivity to $\mathrm{GA}_{3}$ /IAA. SlGRAS7 was upregulated during abiotic stresses following treatment with sodium chloride $(\mathrm{NaCl})$ and D-mannitol in the wild-type (WT) tomato. Tomato plants overexpressing SlGRAS7 showed more resistance to drought and salt stress comparison with WT. Our study of SlGRAS7 in tomato demonstrates how GRAS showed an integrative role, improving resistance to abiotic stresses and enhancing gibberellin/auxin signaling through reproductive as well as vegetative processes.
\end{abstract}

\section{Introduction}

The relationship between molecular developments and environmental clues is measured by the important modifications in gene regulatory networks (GRNs), which play a dynamic part in the manipulability of development and growth in plants [1-3]. Transcriptional regulation, where the transcription factors (TFs) control a succession of target genes in a spatiotemporally particular sequence, is an important member of GRNs [2,4]. Transcription factors from numerous plant varieties show significant functions in stress responses [5], including bZIP, MYC/MYB, ERF, NAC, WRKY, and Dof. The GRAS gene family (named after GAI, RGA, and SCR) is induced through various abiotic stresses $[6,7]$. Transcription factors, such as the GRAS proteins, which are involved in plant growth and pathways of signal transduction, are involved in lateral shoot development [8], phytochrome signaling [9], gametogenesis [10], auxin signaling $[11,12]$, and gibberellin signaling and biosynthesis $[13,14]$.
GRAS proteins are categorized into 13 subfamilies, containing HAM, AtSCR, AtSCL3, AtSCL4/7, AtSCL9, AtSCL28, AtSHR, AtLAS, AtPAT1, Os4, Os19, DELLA, and Pt20 in Arabidopsis, rice, and Populus according to the phylogenetic tree and amino acid sequence alignment [15]. Only a few members of GRAS proteins have been functionally known and involve in signal transduction pathways and plant development. Normally, C-terminus of GRAS proteins has conservative domains, containing PFYRE SAW, VHIID, leucine heptad repeat I (LHR I), and leucine heptad repeat II (LHR II) [16-18]. However, GRAS proteins differ in sequence and length in their $\mathrm{N}$-terminus, which is likely a key element to the functional specificity of each protein [19]. In addition, some experimental evidence confirms that GRAS proteins play vital roles when plants are subject to biotic or abiotic stress. A GRAS transcription factor obtained from Vitis amurensis was used to create transgenic Arabidopsis, and the overexpression of VaPAT1 leads to drought tolerance, high salinity, and cold stress [20]. To identify the 
functions of other GRAS genes in crops, it will help to reveal the pathways that regulate resistance and tolerance to stress and help in the breeding of tolerant species as has been done in tomato plants. Abnormal expression of the PeSCL7 gene enhanced resistance under drought and salt stresses in Arabidopsis [21] and silenced SlGRAS6 plants expressed enhanced tolerance to disease in tomato [22]. In rice, the overexpression of OsGRAS23 increased oxidative and drought stress resistance [23]. In rice and barley, overexpressing miR171 disturbs floral meristem determinacy and phase transitions $[24,25]$. By inhibition of miR156-targeted SPL proteins, the miR171-GRAS component controls trichome distribution and flowering time [26]. This component is also critical for stimulating GA-DELLA signaling in the organization of leaf development in the light and regulation of chlorophyll biosynthesis [27]. In addition, the role of miR171 has been widely studied under several stresses in different varieties, such as maize, barley, Arabidopsis, and potato [28-31]. Thus, many studies showed that GRAS proteins play many significant functions in the tolerance of abiotic or biotic stress.

The actions of the GRAS proteins from DELLA and SCARECROW-like (SCL), gibberellin (GA), and auxin are intimately related to abiotic stress responses and growth processes in plants. Two types of GRAS interact as a complex, AtSCL3 function as a coordinator of SHR-SCR and DELLAs to aid in the cell enlargement of the root endodermis to mediate gibberellin stimulation [32, 33]. AtRGA [13], AtRGL1-3 [14], and AtGAI [34] are DELLA mutants and have been shown to be insensitive to GA. This revealed that increased gibberellin content reduces drought resistance; however, decreased gibberellin content enhances drought resistance [35]. Through a common pathway, the primary participants of gibberellin signaling are DELLA proteins that therefore constrain growth and increase stress resistance [36]. CsSCL1 (Castanea sativa SCL1) in chestnut and PrSCL1 (Pinus radiata SCL1) in pine control adventitious root development through the regulation of auxin signaling [12]. In Arabidopsis, LAX3 and AUX1 are auxin influx carriers, which, combined with the SHR-SCR complex, correlate with lateral and primary root formation [37]. Auxin coordinates the expression of various genes that directly or indirectly respond to stress, and various genes that respond to auxin are controlled through abiotic stresses [38]. In addition, by inducing ROS detoxification enzymes directly or indirectly by affecting the stability of DELLA proteins, it showed that auxin can control ROS homeostasis, which revealed that GA and auxin could coordinate with one another in stress environments $[39,40]$.

Tomato (Solanum lycopersicum) is an important crop because of its great nutritive and commercial value and also a good model plant for fleshy fruit development. However, most GRAS proteins have not been functionally studied in tomato till now. It has been showed that the GRAS family has 53 members in tomato [41]. Overexpression of SlGRAS24 and SlGRAS40 plants showed pleiotropic phenotypes, such as dwarfism, delayed flowering, reduced flower number, and decreased fruit set ratio $[42,43]$. In addition, overexpression of SlGRAS40 enhanced drought and salt tolerance in tomato [42]. By far, there are only seven GRAS proteins that have been functionally studied in tomato, including SIGRAS2, SlGRAS6, SIGRAS24, SIGRAS26, SIGRAS40, SlLs, and SIDELLA. It has been reported that GRAS proteins have multiple functions in many other plant species, so it is important to study the role of other GRAS proteins in tomato, which has not been functionally described yet. Here, we studied the functional description of SlGRAS7 (accession number: Solyc07g065270.1.1), which belongs to a typical PAT1 subfamily gene. To further study the function of SlGRAS7 in tomato, we constructed an overexpression vector to produce SlGRAS7 upregulated transgenic lines. In this study, we found that overexpression of SIGRAS7 resulted in pleiotropic phenotypes and enhanced drought and salt resistance. By evaluating gene expression and hormone responsiveness, we found that alterations in gibberellin and auxin signaling are likely to affect the substandard development of the overexpression of SIGRAS7.

\section{Materials and Methods}

2.1. Plant Growth Conditions. Tomato plants (Solanum lycopersicum cv. Micro-tom) were grown on soil (peat composite: vermiculite, $1: 1)$ in $18 \mathrm{~h}$ light: $6 \mathrm{~h}$ dark cycles, $25^{\circ} \mathrm{C}$ day: $18^{\circ} \mathrm{C}$ night temperatures, and $60 \%$ relative humidity in controlled greenhouse conditions. The plants were treated with water-soluble fertilizers (Stanley Agriculture Group Co. Ltd) weekly. Different tissues from one-month-old WT plants, including leaves, roots, stems, flowers at the anthesis stage, fruits at the immature green, mature green, breaker, breaker plus one day, and orange and red stages, were collected for gene expression analyses. Samples were taken for each tissue from a minimum of seven plants. The different samples from plants were assorted and directly frozen in liquid nitrogen.

2.2. Vector Construction and Plant Transformation. The sequence of SlGRAS7, which does not have a stop codon, was amplified from the tomato cDNA and cloned into an expression vector. Using the standard method [44], Agrobacterium tumefaciens strain GV3101 was prepared to transfer the expression vector using the CaMV 35S promoter. In addition, Agrobacterium tumefaciens was used for transformation into WT tomato plants. Murashige and Skoog (MS) culture medium containing kanamycin was used to screen the positive transgenic lines. Eight SlGRAS7 transgenic overexpression lines (OE) were produced. After qPCR analysis, three of eight homozygous transgenic lines (L1, L2, and L3) in $\mathrm{T} 2$ generation were selected for further experiments.

2.3. Gene Expression Analysis. Total RNA was isolated using an OMEGA BIO-TEK plant RNA kit. The RNA concentration and integrity were measured using a NanoDrop 1000 (Thermo, USA) and agar gel electrophoresis, respectively. First-strand cDNA synthesis was completed using a PrimeScript $^{\mathrm{TM}}$ RT reagent kit with gDNA Eraser (TAKARA, Japan). A Bio-Rad CFX system (Bio-Rad, United States) was used for real time-qPCR with SYBR Green PCR Master Mix (CWBIO, China) in a $25 \mu \mathrm{L}$ total sample volume ( $1 \mu \mathrm{L}$ of primers, $1 \mu \mathrm{L}$ of cDNA, $10.5 \mu \mathrm{L}$ of distilled $\mathrm{H}_{2} \mathrm{O}$, and $12.5 \mu \mathrm{L}$ of $2 \mathrm{x}$ SYBR 
Mix Taq). The RT-qPCR reactions were performed in a 96well iCycler (Bio-Rad), with a temperature program starting with $3 \mathrm{~min}$ at $95^{\circ} \mathrm{C}$, then 40 cycles of $5 \mathrm{sec}$ at $95^{\circ} \mathrm{C}$ and 30 sec at $60^{\circ} \mathrm{C}$. In the end, the melting temperature of the product was determined to verify the specificity of the amplified fragment. Three replicates were conducted for all samples. The SIUBI gene was used as an internal control. Relative expression levels were calculated based on the $2^{-\Delta \Delta C T}$ method. All of the RT-PCR primers are shown in Supplementary Materials (Table S1).

2.4. Hormone Treatment for Plant Growth Analysis. $\mathrm{GA}_{3}$ $(20 \mu \mathrm{M})$ was sprayed on 10-day-old WT and SlGRAS7-OE L2 plants. Both genotypes were sprayed every 2 days for 4 weeks. Control WT and SlGRAS7-OE L2 plants were sprayed with water. The height of the plants and the flowering times for both WT and SIGRAS7-OE L2 were recorded.

Experiments for auxin dose-responses were conducted on one-week-old WT and SlGRAS7-OE L2 seedlings. The hypocotyl section $(8 \mathrm{~mm})$ under the cotyledon nodes was removed. The sections of the hypocotyl were placed in MES buffer/sucrose (5 mM MES/KOH, 1\% $(w / v)$ sucrose, and $\mathrm{pH}$ 6.0) and preincubated for $2 \mathrm{~h}$. Hypocotyl sections were transferred into buffer solutions without or with NAA. After $23 \mathrm{~h}$ of incubation, the hypocotyl sections were measured at room temperature [45].

T2 transgenic lines of SlGRAS7-OE and WT seeds were sterilized, and the SlGRAS7-OE and WT seeds were embedded in the sterilized water for 3 days. The seeds were grown on an MS/2 medium with altered concentrations of $\mathrm{GA}_{3}$ $(0,0.5,10$, and $20 \mu \mathrm{M})$. The seedlings were germinated in the light for $18 \mathrm{~h}$ and the dark for $6 \mathrm{~h}$ in a growth chamber. The day temperature was $25^{\circ} \mathrm{C}$, and the night temperature was $20^{\circ} \mathrm{C}$. The root and shoot lengths and the total plant height were measured after 15 days. Three replicates were performed on 25 plants for each experiment.

2.5. Hormone Treatment for Gene Expression Analysis. In the first experiment, fifteen-day-old WT seedlings were transferred into an MS/2 liquid medium for $0,1,3,6,12$, or 24 h. In the second experiment, fifteen-day-old SlGRAS7-OE L2 and WT seedlings were transferred into an MS/2 liquid medium for $3 \mathrm{~h}$. After treatment, the seedlings from both experiments were transferred into liquid nitrogen and stored at $-80^{\circ} \mathrm{C}$. The control samples were transferred into an $\mathrm{MS} / 2$ liquid medium without hormones. Each experiment was completed with three replicates.

2.6. Abiotic Stress Treatments. Plant leaves of one-month-old WT were sprayed with $100 \mathrm{mM} \mathrm{D}$-mannitol and $200 \mathrm{mM}$ $\mathrm{NaCl}$ to serve as the osmotic and salt stress treatments, respectively. The control WT plants were treated with water. The leaves from the control-, D-mannitol-, and NaCl-treated WT plants were collected after $1 \mathrm{~h}, 3 \mathrm{~h}, 6 \mathrm{~h}, 12 \mathrm{~h}$, and $24 \mathrm{~h}$. Leaves from six plants were collected for each sample and mixed well. Each sample was transferred into liquid nitrogen and stored at $-80^{\circ} \mathrm{C}$ until RNA extraction.

To analyze the salt and drought tolerance, 15 WT plants and 15 plants from each line of SlGRAS7-OE (L1, L2, and L3) were grown in a large pot, and the pots were watered three times a week. The water was constant in all pots. All WT and SlGRAS7-OE plants (L1, L2, and L3) were grown at the same temperature and light conditions. After 2 weeks, the WT and SlGRAS7-OE plants (L1, L2, and L3) were treated as the control, salt, and drought treatments. For the salt treatment, the WT and SlGRAS7-OE (L1, L2, and L3) plants were watered with $200 \mathrm{mM} \mathrm{NaCl}$ at 2-day intervals for one month. The plants were treated without watering to analyze drought. The control plants were treated with water. Light and temperature conditions were the same for all plants treated. The relative water content and total chlorophyll [46] were tested after each treatment. Leaf samples were collected at the same developmental phase after salt and drought treatment, immediately frozen in liquid nitrogen and stored at $-80^{\circ} \mathrm{C}$ until RNA extraction.

For salt and osmotic tolerance analysis, WT and SlGRAS7-OE (L1, L2, and L3) seeds were sterilized and sown on MS/2 alone or MS/2 containing $75 \mathrm{mM} \mathrm{NaCl}$ and $150 \mathrm{mM}$ D-mannitol, respectively [47]. Control WT and SlGRAS7-OE seeds (L1, L2, and L3) were germinated on $\mathrm{MS} / 2$ without $\mathrm{NaCl}$ and $\mathrm{D}$-mannitol. Seeds were germinated in a growth chamber with $18 \mathrm{~h}$ light $\left(25^{\circ} \mathrm{C}\right)$ and $6 \mathrm{~h}$ darkness $\left(18^{\circ} \mathrm{C}\right)$ cycles. The primary roots and hypocotyl lengths were measured after 15 days, and the rate of the seed germination was calculated after one week.

2.7. Statistical Analysis. Each experiment was conducted with three independent biological replicates. Student's $t$-test was used to compare group differences. $P$ values less than 0.05 were considered to be significant.

\section{Results}

3.1. Phenotypic Characterization of the SIGRAS7-OE Transgenic Plants. To evaluate the physiological significance of SlGRAS7, transgenic tomato plants expressing the SlGRAS7 cDNA were produced using a CaMV 35S promoter by transformation with Agrobacterium tumefaciens. WT and overexpressing SlGRAS7 plants are shown in Figure 1(a). Leaves from one-month-old plants of three independent lines, L1, L2, and L3, were found to overexpress the gene by 25.55fold, 30.43-fold, and 23.95-fold, respectively (Figure 1(c)). Overexpression of the SlGRAS7 plants resulted in pleiotropic phenotypes with dwarfism, delayed flowering time, and fewer fruits and seeds. Supplementary Materials (Table S2) show the additional details of the SlGRAS7 phenotypes. SlGRAS7 was expressed in all tissues of the WT examined. SlGRAS7 was expressed at higher levels in the flowers and breaker+1 in the WT (Figure 1(e)).

3.2. SlGRAS7-OE Displays Altered Responsiveness to $G A_{3}$ and $I A A$. The level of expression of SIGRAS7 decreases after treatment with $\mathrm{GA}_{3}$ and IAA in WT (Figure 2(a)), which indicates that SlGRAS7 responds to gibberellin and auxin. To study the functions of SlGRAS7 in response to the phytohormone $\mathrm{GA}_{3}$, WT and SlGRAS7-OE L2 seedlings were germinated with altered concentrations of $\mathrm{GA}_{3}(0 \mu \mathrm{M}, 0.5 \mu \mathrm{M}$, $10 \mu \mathrm{M}$, and $20 \mu \mathrm{M})$. The seedlings that overexpressed 


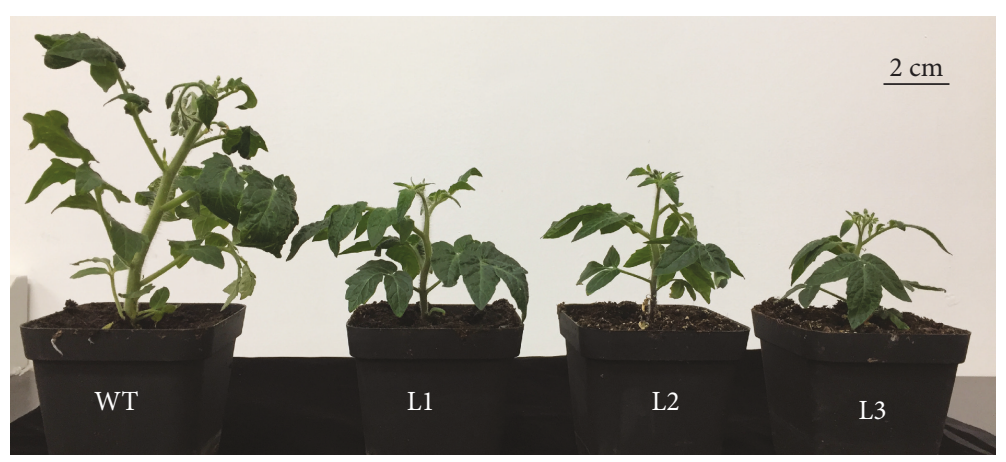

(a)

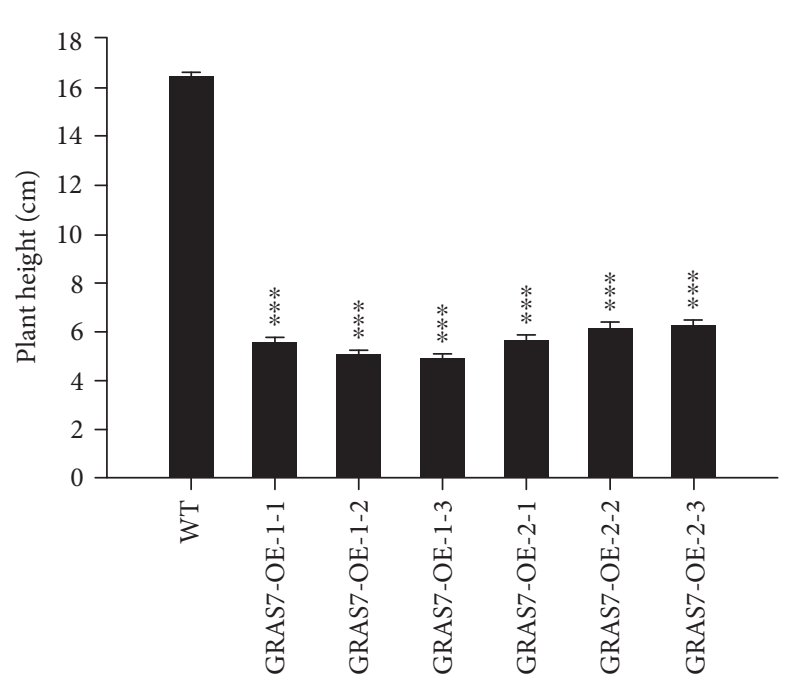

(b)

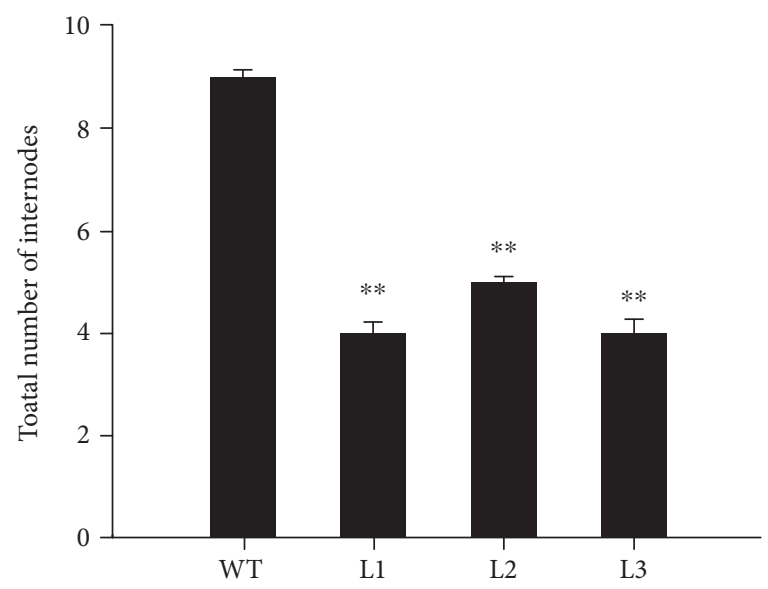

(d)

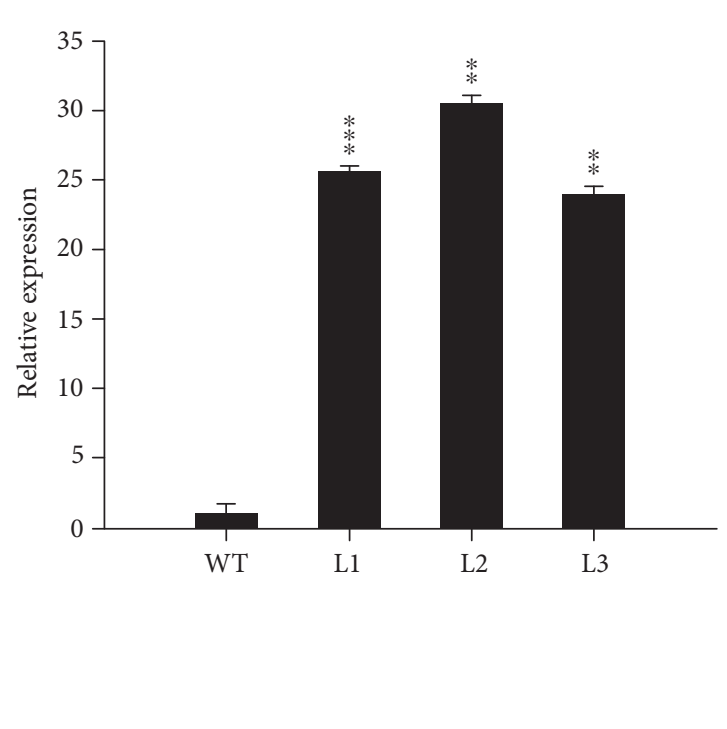

(c)

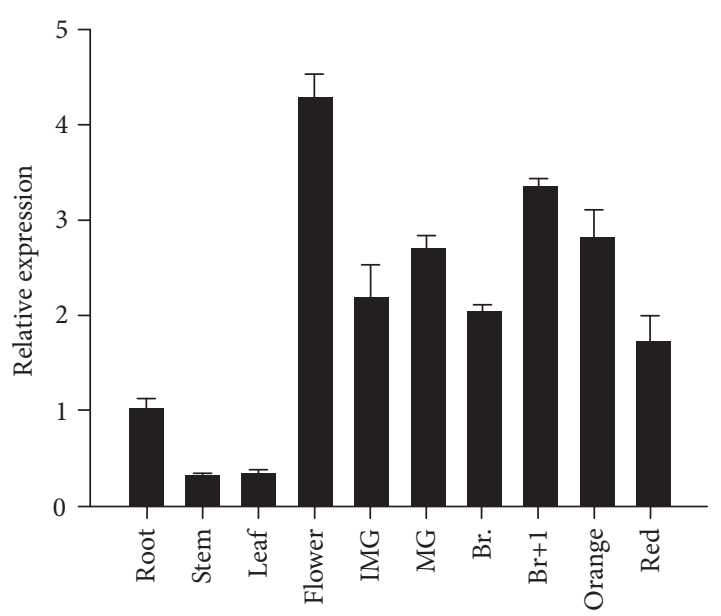

(e)

FIGURE 1: Phenotypic characterization of WT and SlGRAS7-OE. (a) One-month-old plants of WT and transgenic plants of SlGRAS7-OE lines L1, L2, and L3. (b) The height of plants WT and both generations (T1, T2) of SlGRAS7-OE shown in (a). Error bars show the standard error between three biological replicates $(n=3)$ with more than 20 plants for each replicate performed. (c) The expression level of SlGRAS7 of onemonth-old plants of WT and SlGRAS7-OE lines. Expression data were normalized with the SlGRAS7 expression in WT as 1. Error bars show the standard error between three biological replicates $(n=3)$. (d) A total number of internodes of one-month-old plants of WT and SlGRAS7OE (L1, L2, and L3). (e) Tissue profiling analysis of SlGRAS7 in different organs of one-month-old wild-type plants. Expression data were normalized with the SlGRAS7 expression in the root set as 1 . 

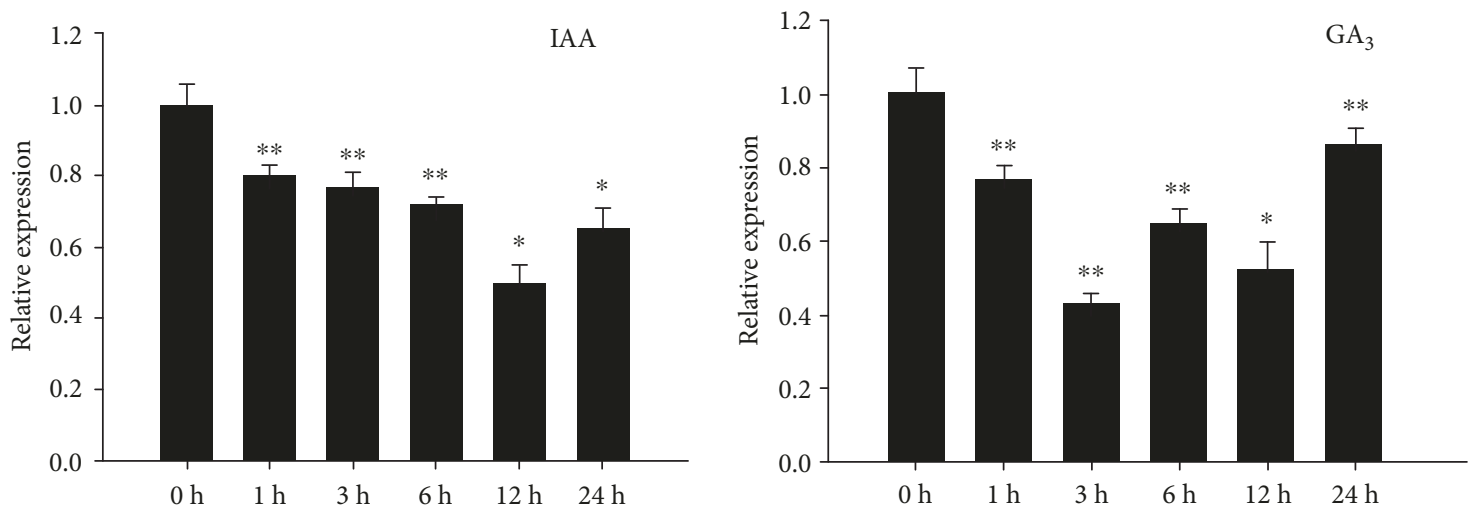

(a)
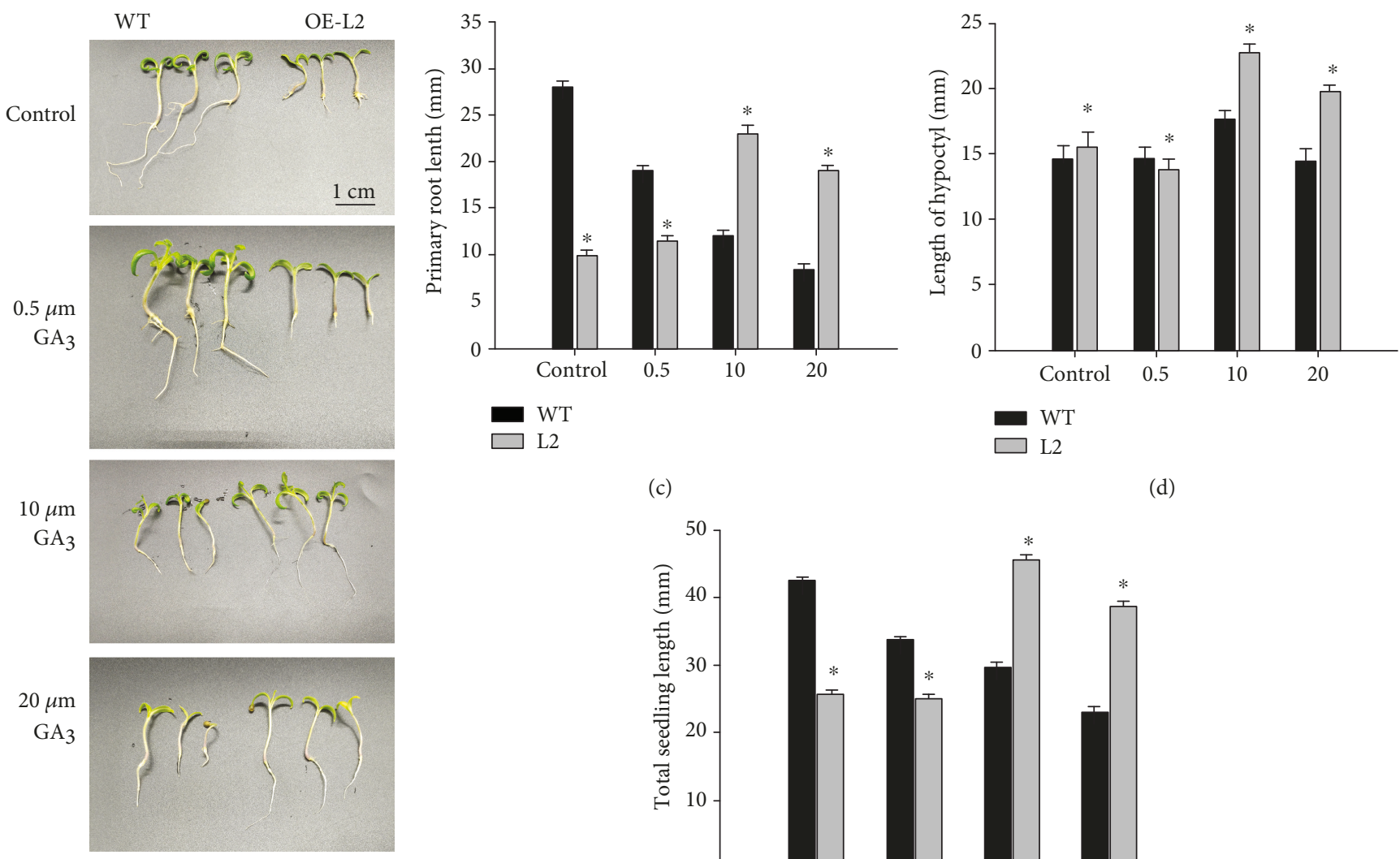

(c)

(d)

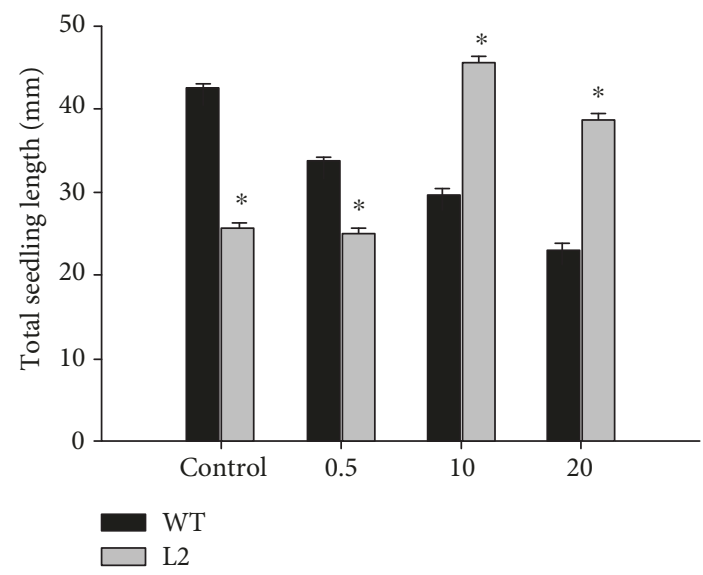

(e)

FIGURE 2: Overexpression of SlGRAS7 alters responsiveness to GA 3 and IAA. (a) Quantitative RT-PCR analysis of $S l G R A S 7$ from 15 days of WT seedlings treated with $20 \mu \mathrm{M} \mathrm{GA}_{3}$ and $20 \mu \mathrm{M}$ IAA. (b) Phenotypes of 15 days of WT and SlGRAS7-OE L2 seedlings grown on an MS/2 medium containing $(0 \mu \mathrm{M}, 0.5 \mu \mathrm{M}, 10 \mu \mathrm{M}$, and $20 \mu \mathrm{M} \mathrm{GA}$ ). (c) Primary root length of WT and SlGRAS7-OE seedlings shown in (b). (d) Hypocotyl length of WT and SlGRAS7-OE seedlings shown in (b). (e) Plant height of WT and SlGRAS7-OE seedlings shown in (b). Asterisks show the significant differences using Student's $t$-test $\left({ }^{*} P<0.05,{ }^{* *} P<0.01\right)$.

SlGRAS7 had no lateral roots and longer primary roots as well as longer hypocotyls than the WT after $\mathrm{GA}_{3}$ treatment (data of L2 in Figures 2(c)-2(e), data of L1 in Figure S1), indicating that overexpression of SlGRAS7 changes the responsiveness to $\mathrm{GA}_{3}$. These data showed that SlGRAS7$\mathrm{OE}$ reduced the responsiveness of the hypocotyl to $\mathrm{GA}_{3}$. The lengths of the roots and hypocotyls of SIGRAS7-OE L2 seedlings were more elongated than those of the WT and SlGRAS7-OE L2 not treated with $\mathrm{GA}_{3}$ in response to $10 \mu \mathrm{M}$ and $20 \mu \mathrm{M} \mathrm{GA}_{3}$. The primary root lengths of SlGRAS7-OE L2 seedlings were smaller than WT seedlings under the control and $0.5 \mu \mathrm{M}$ (Figure 2(b)). The phenotype of the small height and delayed flowering time of SlGRAS7-OE plants could be controlled by exogenous application of 


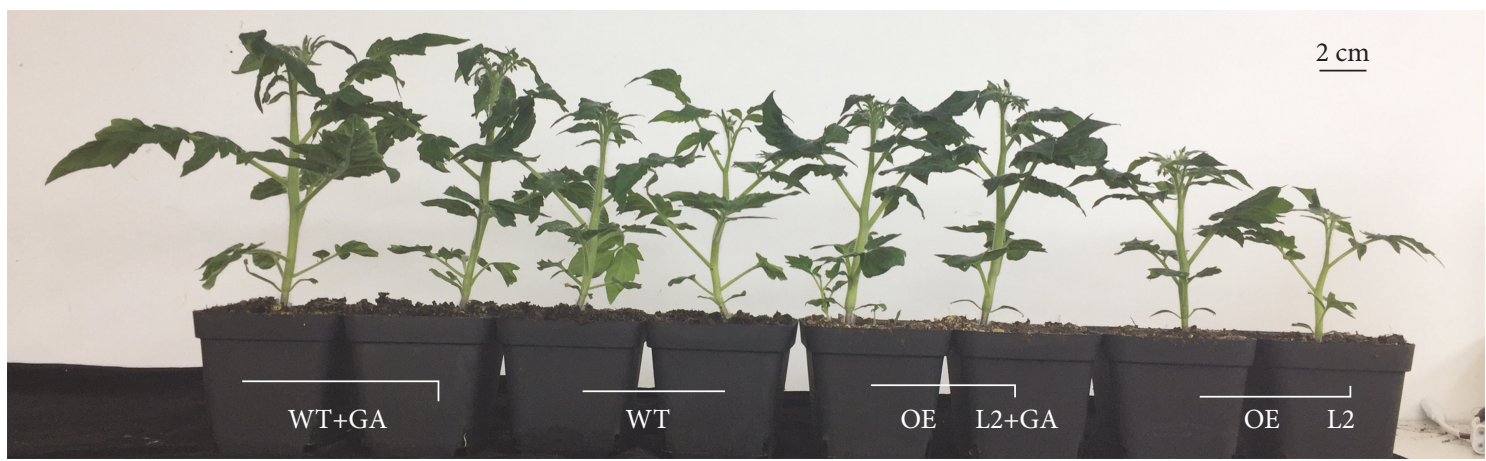

(a)

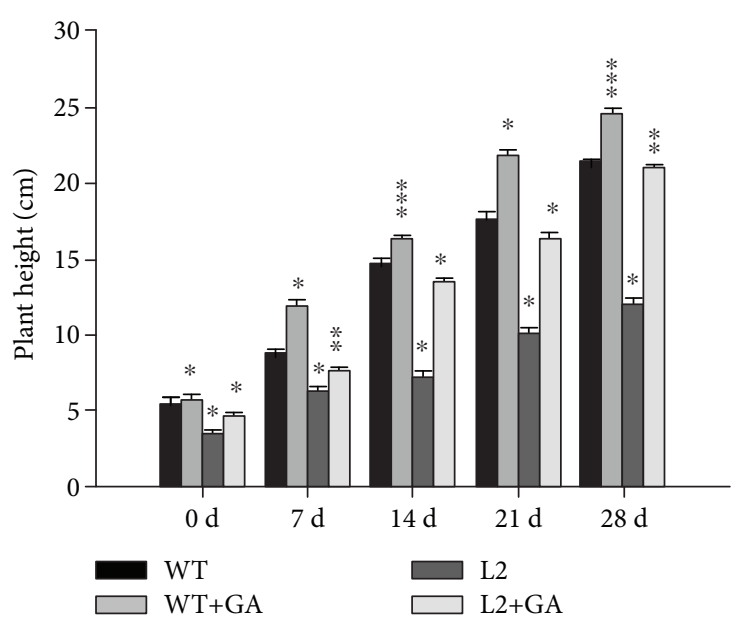

(b)

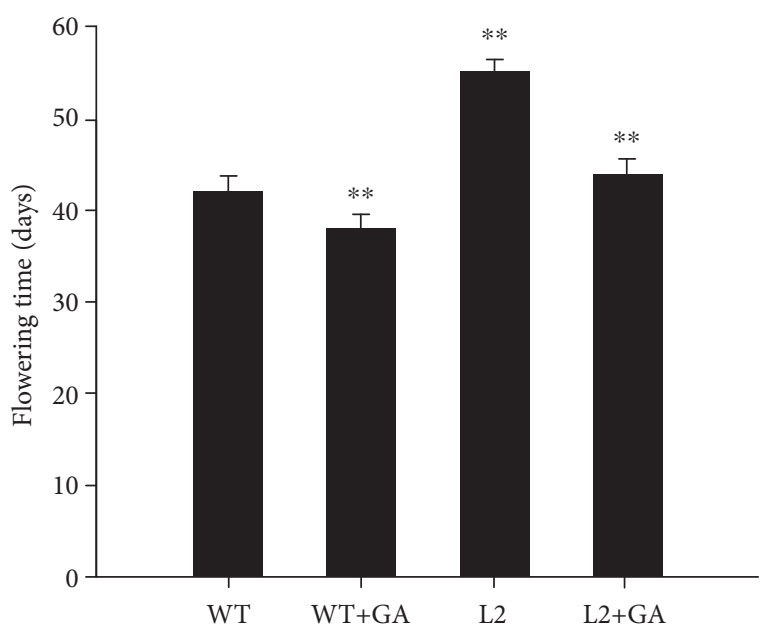

(c)

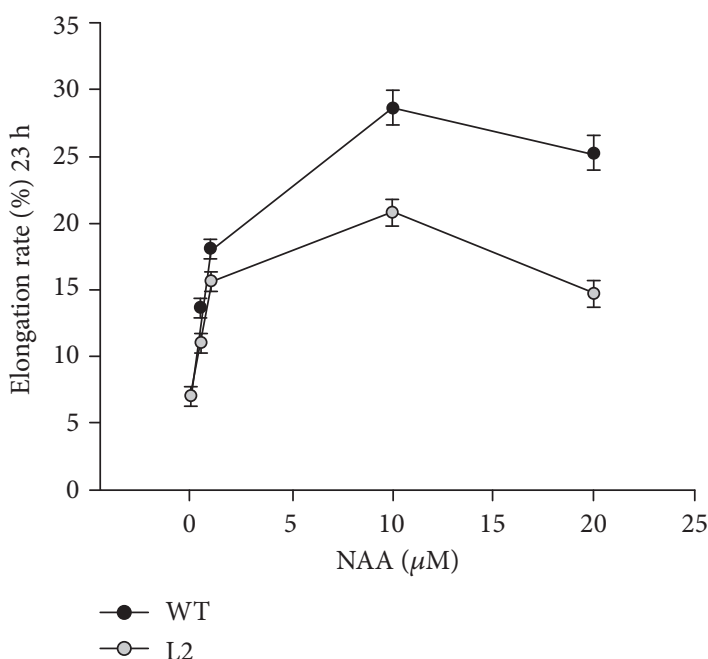

(d)

Figure 3: (a) Rescue of SlGRAS7-OE L2 dwarfism by the exogenous GA $_{3}$ application. (b) Plant height and (c) flowering time of GA -treated $^{-}$ plants shown in (a). (d) Hypocotyl elongation of WT and SIGRAS7-OE L2 after NAA treatment. Asterisks show the significant differences using Student's $t$-test $\left({ }^{*} P<0.05,{ }^{* *} P<0.01\right)$.

$20 \mu \mathrm{M} \mathrm{GA}_{3}$, and their levels became parallel with those of the WT (Figure 3). Thus, the results indicated that SlGRAS7 is involved in GA signaling or biosynthesis. The overexpression of SlGRAS7 showed auxin sensitivity, which was determined by the enlargement of the hypocotyl sections under the auxin dose assay. At all concentrations of auxin, the hypocotyl of SlGRAS7-OE L2 was shorter than that of the WT, but the greatest amount of enlargement of 
the hypocotyl was observed at $10^{-5} \mathrm{M}$ NAA concentrations in the WT, as well as in SlGRAS7-OE L2 (Figure 3(d)). These results showed that overexpression of SlGRAS7 is involved in the reduction of hypocotyl auxin sensitivity.

\subsection{SlGRAS7-OE Enhances Tolerance under Salt and Drought} Stress. WT plants were treated with $\mathrm{NaCl}$ and D-mannitol to show the saline and osmotic effects, respectively. SlGRAS7 was highly upregulated in response to both $200 \mathrm{mM} \mathrm{NaCl}$ and $100 \mathrm{mM}$ D-mannitol stress (Figure 4(a)). Therefore, SlGRAS7 may be involved in the abiotic and osmotic stress response in tomato.

To explore the role of SIGRAS7 under salt and drought stress, WT plants and SlGRAS7-OE L1, L2, and L3 were treated with a solution of $200 \mathrm{mM} \mathrm{NaCl}$ every $48 \mathrm{~h}$ for up to one month to examine salt stress tolerance. WT and SlGRAS7-OE L1, L2, and L3 plants were deprived of water for up to one month to examine drought stress. Under $\mathrm{NaCl}$ salt stress and drought stress treatment, all SlGRAS7-OE plants were healthier than the WT (Figure 4(b)). After one month, WT plants generally displayed more necrosis and chlorosis under salt stress treatment, while there was no apparent damage to SlGRAS7-OE plants (Figure 4(b)). Under drought stress treatment, the lower leaves wilted more in WT plants, but the only insignificant damage was noted in SlGRAS7-OE plants (Figure 4(b)).

During salt and drought stress treatments, both the relative water content (RWC) and the total chlorophyll content reduced in WT and SIGRAS7-OE plants, while the levels of both were much greater in SlGRAS7-OE plants than in WT (Figures 4(c)-4(f)).

3.4. SIGRAS7-OE Enhances the Seed Germination Rate under Salt and Osmotic Stress. WT and SlGRAS7-OE L1, L2, and L3 plants were examined to determine the salt and osmotic tolerance of seed germination (Figure 5(a)). The rate of seed germination of both WT and SlGRAS7-OE decreased in response to $75 \mathrm{mM} \mathrm{NaCl}$ and $150 \mathrm{mM} \mathrm{D}$-mannitol, respectively (Figure 5(a)), but the rate of seed germination of SIGRAS7-OE was much greater than that of WT seeds in response to both stress treatments. The average rate for the salt treatment was $65 \% / 73.8 \%$ and $76.7 \% / 72.4 \%$ for the osmotic treatment. Root elongation was affected in response to salt and osmotic stress treatments, and the root length of SlGRAS7-OE was longer than that of the WT under the salt and osmotic treatments (Figure 5(c)). The shoot lengths of SIGRAS7-OE were larger than those of the WT under both stress treatments (Figure 5(d)). The root and shoot lengths of WT were significantly reduced under both stress treatments. These results indicate that the seeds and seedlings of SIGRAS7-OE tolerate salt and osmotic stress.

3.5. Expression Analysis of Auxin- and GA-Related Genes in SlGRAS7-OE Plants. To examine the role of SlGRAS7 in the auxin and GA pathways, the levels of expression of 21 tomato genes were tested in WT seedlings and those of SlGRAS7-OE $\mathrm{L} 2$ under auxin and $\mathrm{GA}_{3}$ treatments (Figure 6). Four PINFORMED (PIN) auxin efflux transport proteins (SIPIN1, SlPIN3, SlPIN5, and SlPIN6), four auxin response gene
(ARF) transcription factors (SlARF5, SlARF6, SlARF7, and SlARF8), three GA deactivating enzymes (SlGA2ox1, SlGA2ox2, and SlGAox4), four indole-3-acetic acid/auxin (IAA/Aux) transcription factors (SlIAA3, SlIAA4, SlIAA7, and SIIAA9), a key regulator of the GA signaling pathway (SIDELLA), and five GA biosynthetic enzymes (SlGA20ox1, SlGA20ox2, SlGA20ox4, SlGA3ox1, and SlGAox2) were examined to determine their expression levels in the WT and SlGRAS7-OE. In the control, nine genes showed higher expression and 12 showed lower expression in the SlGRAS7-OE, which indicated that overexpression of SlGRAS7 altered auxin and GA homeostasis in overexpressing plants. In addition, when these results were compared to WT, some genes showed different responses to IAA and $\mathrm{GA}_{3}$ in SlGRAS7-OE seedlings. For example, the expression of SIAFR5 is downregulated in the WT but upregulated in the SlGRAS7-OE seedlings in response to IAA treatment. SIPIN6 was upregulated by IAA and GA $_{3}$ in the WT and SIGRAS7-OE. SIDELLA was downregulated by IAA but upregulated by $\mathrm{GA}_{3}$ in the WT, but it was upregulated under the IAA treatment and downregulated under the $\mathrm{GA}_{3}$ treatment in SlGRAS7-OE. $\mathrm{GA}_{3}$ induces the upregulation of SlGA2ox4 in both WT and SIGRAS7-OE. Without hormone treatment, the expression of SlGA20ox1 was upregulated in SlGRAS7-OE seedlings, but IAA treatment caused the downregulation of expression. WT and SlGRAS7-OE seedlings displayed a dramatic response to the IAA-related genes during $\mathrm{GA}_{3}$ treatment. In contrast, the GA-related genes during IAA treatment could indicate that SlGRAS7 acts as an integrator between the auxin and GA pathways. However, it is likely that SIGRAS7 has a role in the regulation of hormone-related gene expression in tomato, primarily in the genes related to auxin and GA transport, biosynthesis, and signal transduction.

3.6. Expression Analysis of Stress-Related Genes in WT and SlGRAS7-OE Plants under Salt and Drought Stress. Quantitative reverse transcription- (qRT-) PCR was used to examine the expression of plant stress response biomarkers to determine the molecular mechanisms involved in the enhanced resistance of SlGRAS7-OE in response to salt and drought stress (Figure 7). Scavenging and ROS generation alter the transcript levels of many genes involved, such as CAT, $P O D, S O D$, ascorbate peroxidase $(A P X)$, glutathione $\mathrm{S}$ transferase (GST), and lipoxygenase (LOX). These were measured under normal and stress conditions in both WT and SlGRAS7-OE. In SlGRAS7-OE plants, the expression levels became higher than those in WT under stress conditions (Figure 7). An ascorbic acid synthetase gene (SlGME2) showed upregulation in response to the control and stress conditions in SlGRAS7-OE plants compared to WT, and the level of SIGEM2 also increased under stress conditions in WT plants (Figure 7). A heat shock protein (SlHsp90-1) had higher levels of SlGRAS7-OE plants than in WT after salt and drought treatment (Figure 7). An ethyleneresponsive factor (SIERF1), an ethylene-responsive LEA protein (SlERF5), and an ascorbate peroxidase gene (SlAPX) all had higher levels in the SlGRAS7-OE plants under control and salt conditions compared to that in the WT (Figure 7). 

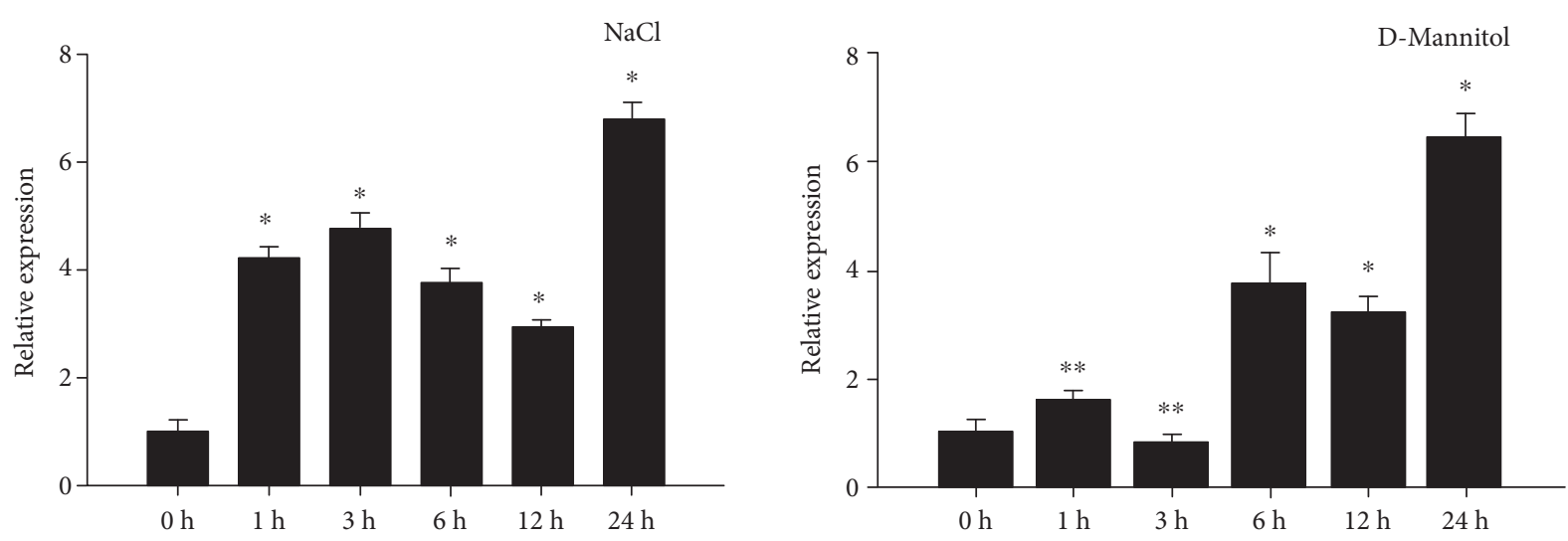

(a)
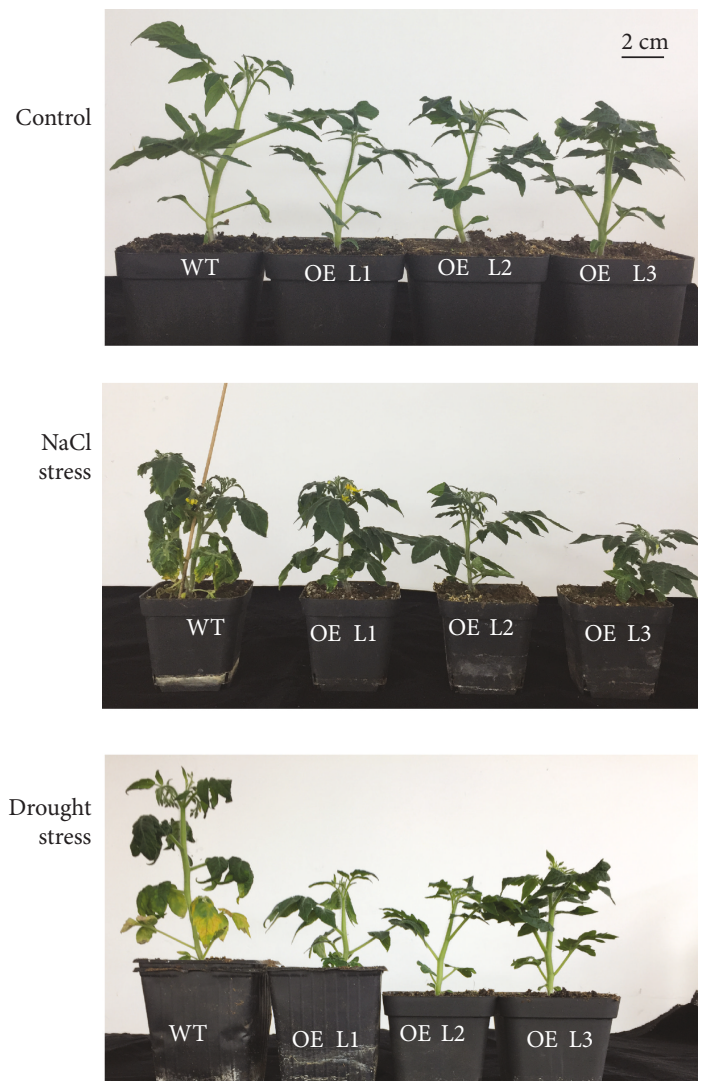

(b)

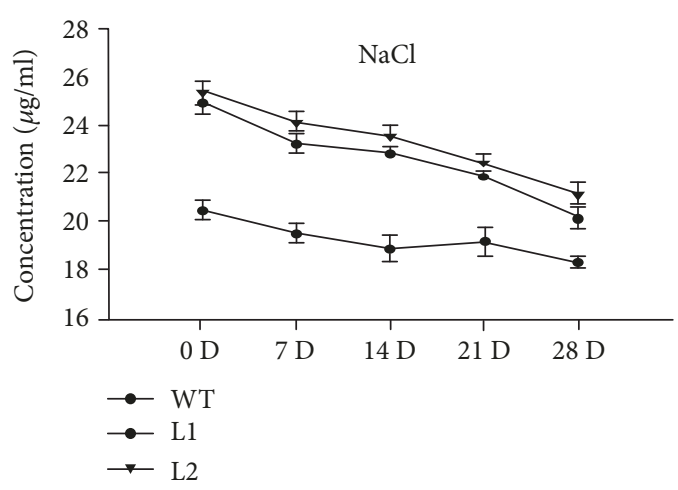

(c)

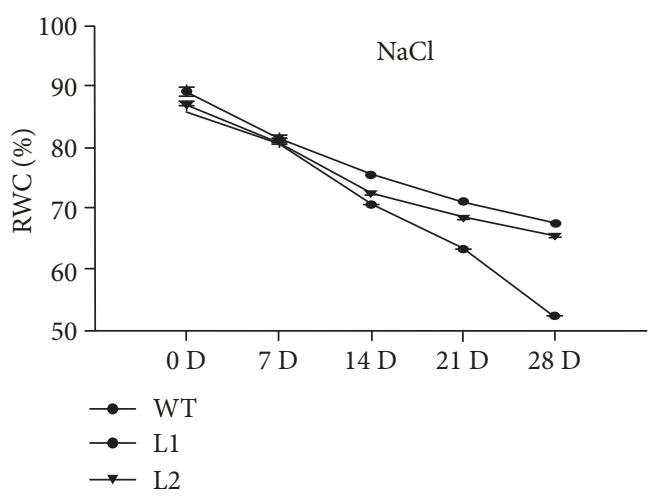

(d)

Figure 4: Continued. 


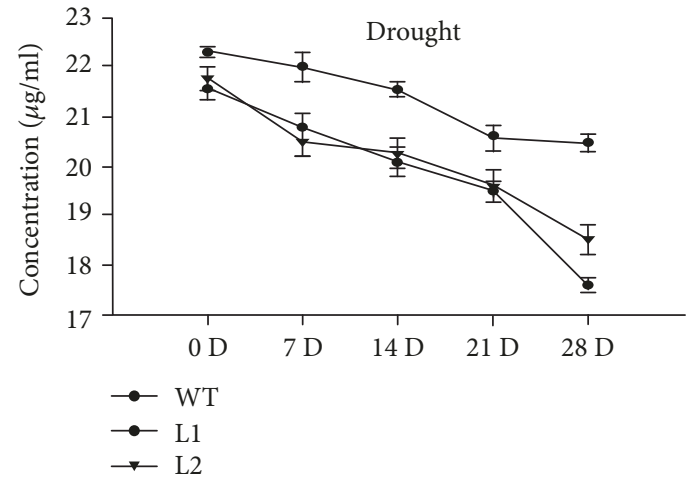

(e)

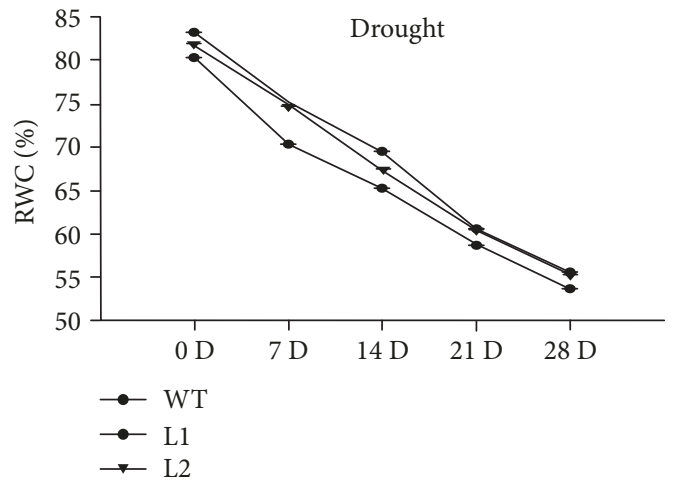

(f)

FIGURE 4: Overexpression of SlGRAS7 enhances tolerance to salt and drought stress treatment. (a) Quantitative RT-PCR analysis of SlGRAS7 from one-month-old plants sprayed with $200 \mathrm{mM} \mathrm{NaCl}$ and $100 \mathrm{mM} \mathrm{D-mannitol.} \mathrm{Expression} \mathrm{data} \mathrm{were} \mathrm{normalized} \mathrm{with} \mathrm{an} \mathrm{expression} \mathrm{of}$ SlGRAS7 in treated plants as $0 \mathrm{~h}$ set as 1 . Asterisks show significant differences using Student's $t$-test $\left({ }^{*} P<0.05\right.$, $\left.{ }^{* *} P<0.01\right)$. (b) Photographs of representative plants after one month of $\mathrm{NaCl}$ salt treatment and of drought stress compared to control plants. (c, d) Total chlorophyll concentration and relative water content (RWC) of plants shown in (b) under salt stress. (e, f) Total chlorophyll concentration and relative water content (RWC) of plants shown in (b) under drought stress.

SlCAT2 was upregulated after control and drought conditions in both SlGRAS7-OE plants and WT plants, but after salt stress, the transcript level decreased more in SlGRAS7OE plants than in WT plants (Figure 7). These results showed that SlGRAS7 could play an important role in stress signaling pathways by modifying these genes in tomato.

\section{Discussion}

Mounting evidence shows that GRAS transcription factors play dynamic roles in plant development and signal transduction pathways. A comprehensive studied miR171-GRAS control network takes part in complex physiological developments, such as shoot branching, shoot meristem maintenance, trichome distribution, chlorophyll biosynthesis, and flowering time $[26,27,43,48]$. Recently, its similar regulatory module has been studied in tomato [42, 43]. Overexpression of SlGRAS40 enhances tolerance to abiotic stresses and influences gibberellin and auxin pathway during reproductive and vegetative growth in tomato [42], and overproduction of a tomato miR171 target gene SlGRAS24 impacts several agronomical behaviors through regulating auxin and gibberellin homeostasis [43]. Downregulation of SlGRAS26 altered plant phase transition and morphological traits in tomato. SlGRAS26 showed a response to ABA, GA, IAA, dehydration, and abiotic stresses [49]. There are eleven GRAS proteins that belong to the PAT1 subfamily. However, the function of PAT1 branch has never been described so far in tomato. Here, one gene from the PAT1 subfamily, SlGRAS7, was functionally recognized. SlGRAS7 showed enhance resistance to abiotic stresses and hormone treatments. These results indicated that SlGRAS7 may be involved in the abiotic stress responsive and mediating hormone signaling.

Salt and drought stress can lower metabolic reactions, reduce photosynthetic capacity, and enhance the oxidative loss of cells [50]. Due to salt and drought stress, indications of damage to the plants, such as necrosis, chlorosis, and wilting, were all delayed in overexpressing SlGRAS7 tomato plants compared to WT tomato plants (Figure 4(b)). The concentration of total chlorophyll and the relative water content were higher in SlGRAS7-OE plants than in WT (Figures 4(c)-4(f)). Under $\mathrm{NaCl}$ and D-mannitol stress treatments, the germination rates of seeds and seedlings were less affected in SlGRAS7-OE than in WT (Figure 5(b)). The results showed that SlGRAS7-OE increased the ability to resist salt and drought stress during vegetative growth. Several genes have been reported to be upregulated in the vegetative tissues in response to stress treatments [51, 52]. In this study, the transcription levels of numerous genes have been confirmed to affect ROS scavenging (Figure 7). Under control and stress treatments, the expression of SISOD, SlLOX, SlGST, SlCAT2, and SlAPX increased during the overexpression of SlGRAS7 compared to the WT (Figure 7). SlERF1, a key factor of biotic/abiotic stress responses [53], and SlERF5 showed higher levels of expression in SlGRAS7$\mathrm{OE}$ under salt and drought stress conditions compared to WT plants (Figure 7). SlGME2, an important catalytic enzyme in the biosynthesis of ascorbic acid [54], and heat shock protein (SlHsp90-1) both showed higher expression under salt and drought stress. These results indicated that SlGRAS7-OE modifies gene expression involved in stress signaling pathways, which could be a mechanism to increase salt and drought stress tolerance. In contrast, SlGRAS7-OE resulted in the adaptation of various significant agronomical behaviors, including plant height, stem length, stem diameter, leaf length, leaf diameter, and flowering time (Supplementary Table S2), which makes it a good target gene to produce varieties with differing plant architectures and flowering times that result in altered yields. Thus, this could result in varieties that help achieve the maximum demand for nutrition, feed, and biofuel production.

Gibberellin and auxin participate in abiotic stress responses in plants. For instance, ABA signaling and saltactivated ethylene pathways integrate at the level of DELLAs 


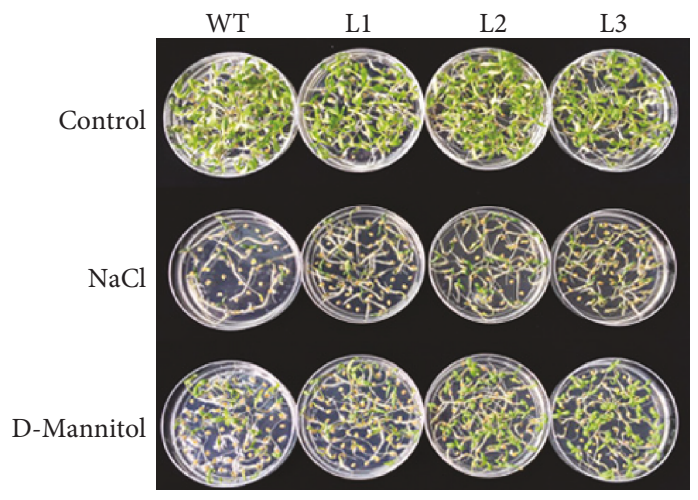

(a)

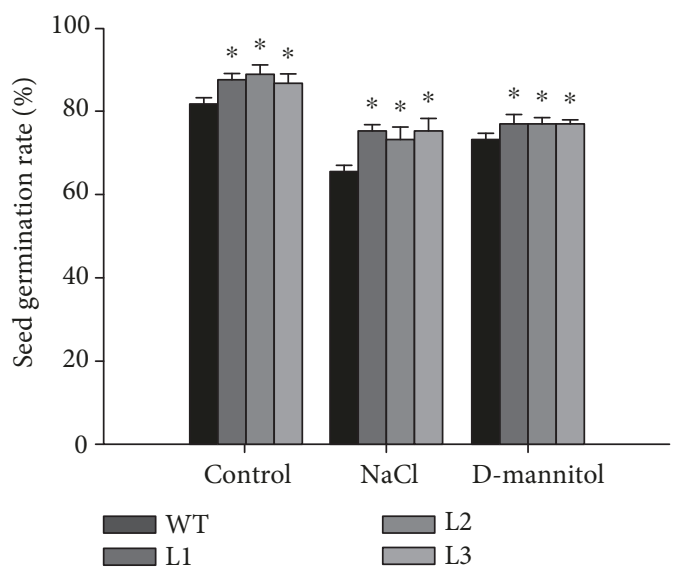

(c)

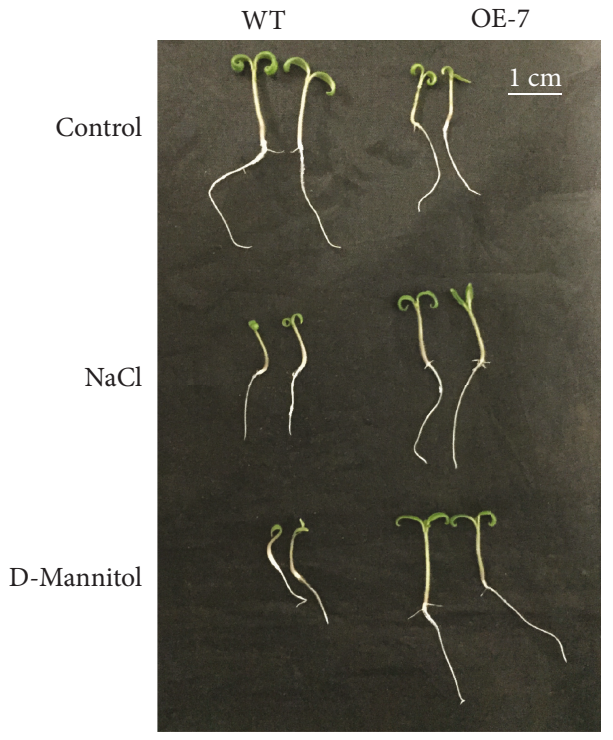

(b)

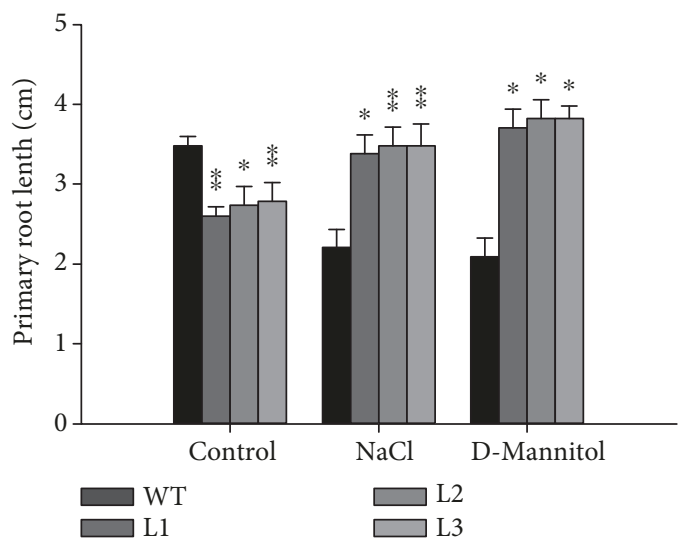

(d)

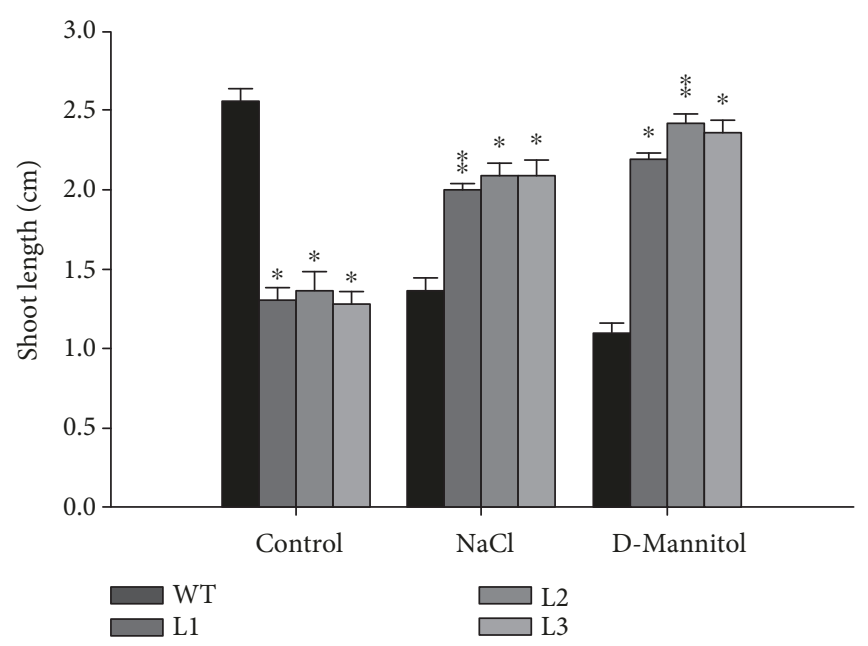

(e)

Figure 5: Comparative analysis of SlGRAS7-OE under salt and osmotic stress. (a, b) Seed germination of WT and SlGRAS7-OE under salt and D-mannitol stress treatments after 2 weeks. (c) The germination rate of WT and SlGRAS7-OE lines under control, salt, and osmotic stress. (d) Primary root length of WT and SlGRAS7-OE under control, NaCl, and D-mannitol treatments. (e) Shoot lengths of WT and SlGRAS7-OE under control, $\mathrm{NaCl}$, and $\mathrm{D}$-mannitol treatments. Error bars indicate the standard errors between three replicates. Asterisks show significant differences using Student's $t$-test $\left({ }^{*} P<0.05,{ }^{* *} P<0.01\right)$. 

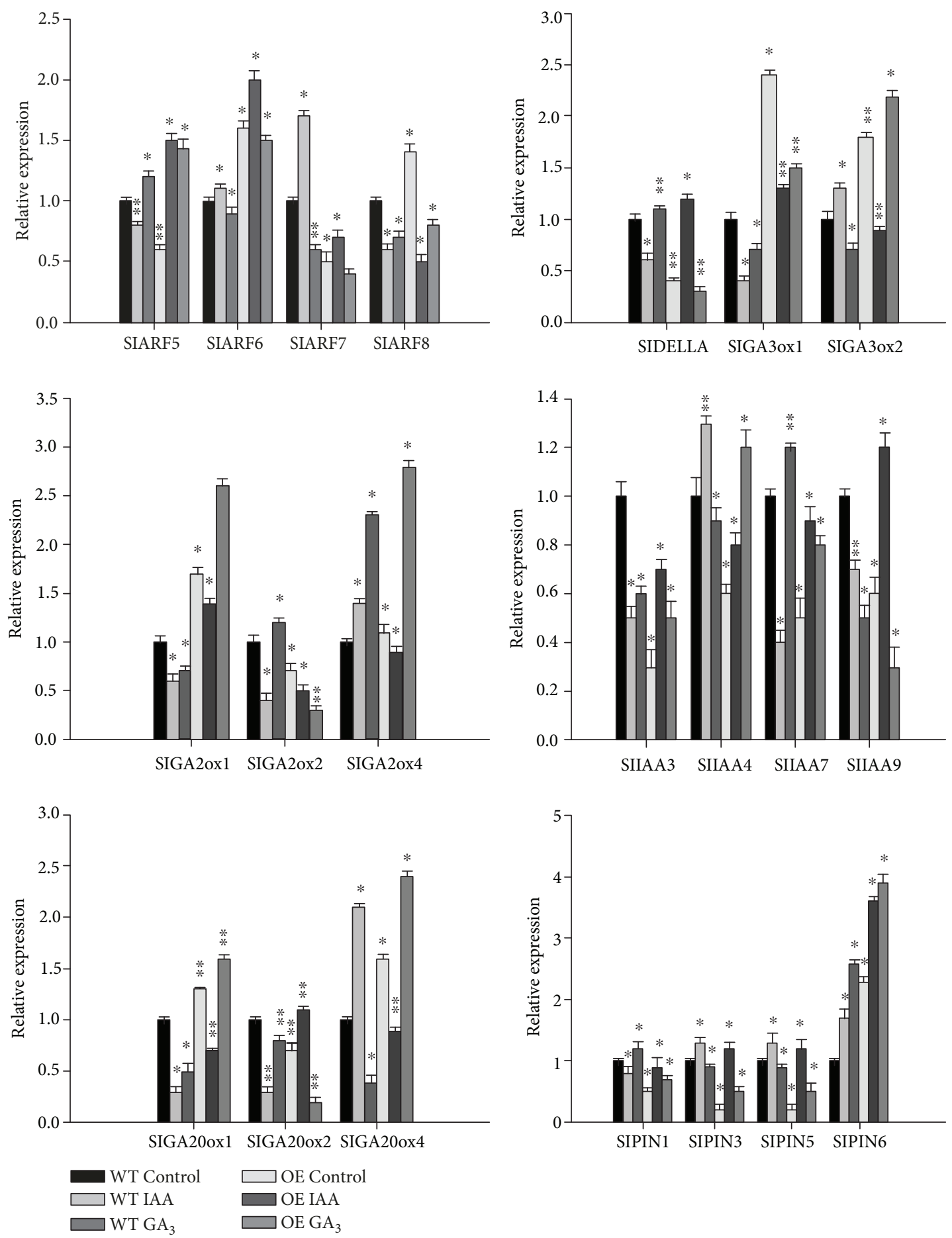

FIGURE 6: Expression analysis of auxin/GA-related genes. qRT-PCR analysis of auxin and GA-related genes in 15 days old WT and SlGRAS7$\mathrm{OE}$ seedlings as well as in response to IAA and $\mathrm{GA}_{3}$ treatment $(20 \mu \mathrm{M}$ for $3 \mathrm{~h})$. Error bars represent the standard error between three biological replicates performed. Asterisks show significant differences using Student's $t$-test $\left({ }^{*} P<0.05,{ }^{* *} P<0.01\right)$.

to enhance salt resistance [55]. Under abiotic stress, DELLA proteins are also involved in the regulation of growth and ROS reactions [36]. Mannitol and salt stress treatments can boost the accretion of DELLAs with upregulation of the genes encoding antioxidant mechanisms, supersede with the cutback in ROS abundance $[55,56]$. An association has been found between abiotic stress and endogenous auxin levels in rice in which overexpression of OsGH3.13 or OsPIN3t enhanced resistance under drought $[57,58]$. Various studies also showed that there is a correlation between ROS and auxin pathways. The exogenous application of auxin reduced the $\mathrm{H}_{2} \mathrm{O}_{2}$ content in the roots of the tomato 

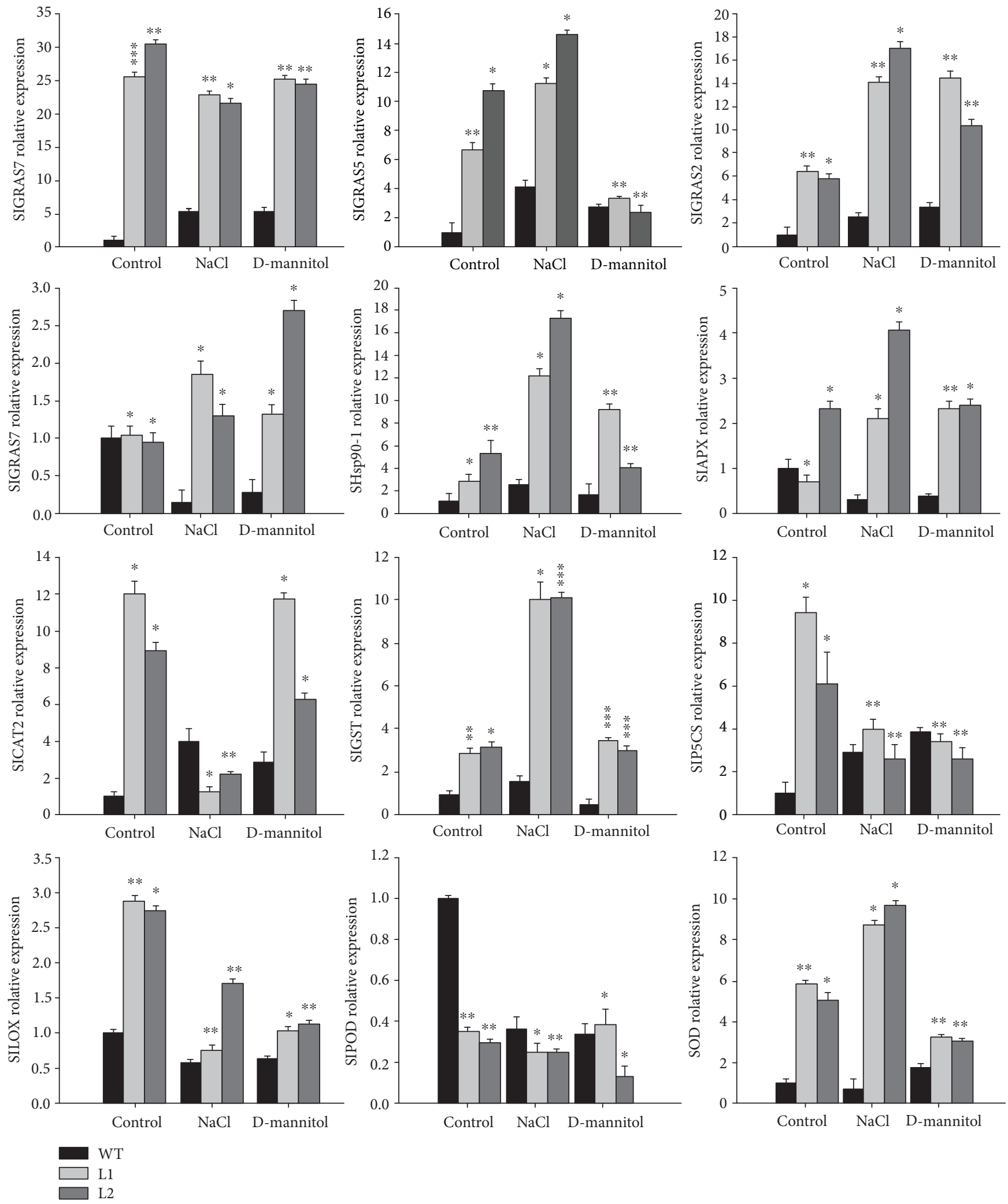

Figure 7: Expression levels of stress-related genes (ERF1, ERF5, GME2, Hsp90-1, P5CS APX, CAT2, GST, LOX, POD, and SOD) in WT and SlGRAS7-OE (L1, L2) under normal, $\mathrm{NaCl}$, and D-mannitol. Error bars show the standard error of data. Asterisks show the significant differences using Student's $t$-test $\left({ }^{*} P<0.05,{ }^{* *} P<0.01\right)$. 
by enhancing the activity and expression of $\mathrm{H}_{2} \mathrm{O}_{2}$ scavenging enzymes [59].

Several studies showed that GAs control various developmental and growth processes, for instance, stem elongation [60]. An ERF/AP2 transcription factor, overexpression of $S I D R E B$, shows small heightened tomato plants by inhibiting gibberellin biosynthesis to decrease endogenous gibberellin level [61]. Overexpression of AtGA20ox1, AtGA20ox2, and AtGA20ox3 augmented shoot growth and exhibited elongated hypocotyls by producing more dynamic GAs in Arabidopsis [34, 62]. Overexpression of the CcGA20ox1 gene in tomato also has similar phenotypes [63]. Moreover, GRAS proteins have been reported in association with GA regulation. For instance, GRAS protein SCL3 and DELLA antagonize each other in controlling both downstream GA responses and upstream GA biosynthetic genes [33]. The association of GRAS proteins and GA has been widely known [19]. However, some proteins from the GRAS family have also been known to be involved in auxin signaling. For example, AtSCL15 is an auxin-induced GRAS protein involved in seed maturation [64]. By modulating both auxin and GA signaling, the SHR/SCR complex has been shown to participate in root growth $[32,33,37]$. The relation between auxin and GA has been clearly shown by the identification of crosstalk and self-regulatory pathways, including genes related to GA metabolism and auxin transport. However, numerous significant characteristics of this relationship are still unknown. Plant hormones function in the development and growth of root apical meristems (RAMs) and shoot apical meristems (SAMs) [65-67]. Auxin/GA signaling roles have been recognized in RAMs and SAMs [68]. It has been shown that HAM genes are necessary to sustain both RAMs and SAMs [69], indicating that these genes could play roles in RAMs and SAMs by controlling auxin and GA signaling. While Atham1, 2, 3 is known to produce root apex auxin maxima that are related to the WT in intensity and spatial expression in Arabidopsis [69], it did not directly correlate with the action of the AtHAM gene to auxin signaling. In this study, we show that SIGRAS7 is an important transcription factor that may be involved in auxin and GA signaling pathways. It is also known that a GRAS-like gene of sunflower altered the gibberellin content and axillary meristem outgrowth of transgenic Arabidopsis plants [70]. The overexpression of SlGRAS7 in tomato plants resulted in a dwarf phenotype that has small primary roots, short stem lengths, and later flowering time (Supplementary Table S2). Some GRAS has been shown to function as regulators of gibberellin and auxin in the development and growth of the plant. It has been described how SIGRAS24 influenced a number of agronomical behaviors in tomato by regulating gibberellin and auxin homeostasis [43]. In our study, overexpression of SlGRAS7 altered the responsiveness to $\mathrm{GA}_{3}$ and IAA (Figure 2), which leads to a shortage of GA, auxin insensitivity, and an altered abundance of transcripts linked to gibberellin and auxin signaling and biosynthesis (Figure 6). Some of the GA-related genes were downregulated, indicating that the GA content was disrupted in SlGRAS7-OE (Figure 6). In addition, the application of $\mathrm{GA}_{3}$ partially inhibited the dwarf phenotype and the growth rate to the WT level in SlGRAS7-OE plants (Figures 3(a)-3(d)). These results suggested that SlGRAS7 may be involved in GA and auxin signaling. Our results also indicated that SlGRAS7 may enhance the abiotic stress response via GA/auxin signaling in tomato plants. SlGRAS7 disturbs auxin signaling and represses gibberellin biosynthesis by reducing gene expression encoding auxin transporters and receptors, and GA biosynthesis stimulating enzymes, respectively, then affects auxin and gibberellin homeostasis. Consequently, we conclude that overexpressing SlGRAS7 plants may enhance the abiotic tolerance and ROS scavenging ability. Also, the crosstalk between gibberellin and auxin may stimulate DELLA accumulation under abiotic stresses in SlGRAS7-OE plants. Thus, our studies on SlGRAS7 shows that GRAS may play an integrative function in tomato and may enhance tolerance to abiotic stresses, gibberellin, and auxin signaling during reproductive and vegetative growth.

\section{Abbreviations}

GRAS: $\quad$ Gibberellic acid insensitive, repressor of GA1, and scarecrow

WT: Wild-type

SIGRAS7-OE: Overexpression of SIGRAS7

GRNs: Gene regulatory networks

TF: Transcription factors

LHR: $\quad$ Leucine heptad repeat

GA: Gibberellin

IAA: Indole acetic acid

cDNA: DNA complementary to RNA

RNA: Ribonucleic acid

RT-qPCR: Real-time quantitative PCR

FAA: $\quad$ Formalin-acetic-alcohol

bZIP: $\quad$ Basic-domain leucine-zipper

MYC/MYB: Myelocytomatosis/myeloblastosis

NAC: $\quad$ N-Acetylcysteine

Dof: DNA-binding with one finger

LOM: $\quad$ Lost meristem

SPL: $\quad$ Squamosa-promoter binding protein-like

SCL: $\quad$ Scarecrow-like

MES: $\quad$ 2-(N-morpholino) ethanesulfonic acid

KOH: $\quad$ Potassium hydroxide

MS: $\quad$ Murashige and Skoog

$\mathrm{NaCl}$ : Sodium chloride

RWC: $\quad$ Relative water content

ROS: $\quad$ Reactive oxygen species

ABA: Abscisic acid

ARF: $\quad$ Auxin response factor

IAA/Aux: Indole-3-acetic acid/auxin

CAT: $\quad$ Catalase

POD: $\quad$ Peroxidase

SOD: $\quad$ Superoxide dismutase

APX: $\quad$ Ascorbate peroxidase

GS: Glutathione S-transferase

LOX: Lipoxygenase

GME: GDP-mannose $3{ }^{\prime}, 5{ }^{\prime}$-epimerase

Hsp: Heat shock protein

ERF: $\quad$ Ethylene-responsive factor

LEA: Late embryogenesis abundant 


$\begin{array}{ll}\text { APX: } & \text { Ascorbate peroxidase } \\ \mathrm{H}_{2} \mathrm{O}_{2}: & \text { Hydrogen peroxide } \\ \text { SHR/SCR: } & \text { Short root/scarecrow } \\ \text { RAM: } & \text { Root apical meristem } \\ \text { SAM: } & \text { Shoot apical meristem } \\ \text { HAM: } & \text { Hairy meristem. }\end{array}$

\section{Data Availability}

The data used to support the findings of this study are included within the article.

\section{Conflicts of Interest}

All authors declared that there is no conflict of interest.

\section{Acknowledgments}

This work was supported by the National Natural Science Foundation of China (31572175) and the Chongqing Science and Technology Commission (CSTCKJCXLJRC15).

\section{Supplementary Materials}

Supplementary Table S1: primers used for qRT-PCR. Supplementary Table S2: phenotypes of WT and SlGRAS7-OE plants. Supplementary Figure S1: 15 days of WT and SlGRAS7-OE L2 seedlings grown on an MS/2 medium containing $\left(0 \mu \mathrm{M}, 0.5 \mu \mathrm{M}, 10 \mu \mathrm{M}\right.$, and $\left.20 \mu \mathrm{M} \mathrm{GA}_{3}\right)$. (a) Primary root length of WT and SlGRAS7-OE (L1) seedlings. (b) Hypocotyl length of WT and SIGRAS7-OE (L1) seedlings. (c) Plant height of WT and SlGRAS7-OE seedlings (L1). Asterisks show the significant differences using Student's t-test $\left({ }^{*} P<0.05,{ }^{* *} P<0.01\right)$. (Supplementary Materials)

\section{References}

[1] A. Gaudinier and S. M. Brady, "Mapping transcriptional networks in plants: data-driven discovery of novel biological mechanisms," Annual Review of Plant Biology, vol. 67, no. 1, pp. 575-594, 2016.

[2] K. Kaufmann, A. Pajoro, and G. C. Angenent, "Regulation of transcription in plants: mechanisms controlling developmental switches," Nature Reviews Genetics, vol. 11, no. 12, pp. 830-842, 2010.

[3] H. Wolters and G. Jürgens, "Survival of the flexible: hormonal growth control and adaptation in plant development," Nature Reviews Genetics, vol. 10, no. 5, pp. 305-317, 2009.

[4] U. Alon, "Network motifs: theory and experimental approaches," Nature Reviews Genetics, vol. 8, no. 6, pp. 450461, 2007.

[5] K. B. Singh, R. C. Foley, and L. Oñate-Sánchez, "Transcription factors in plant defense and stress responses," Current Opinion in Plant Biology, vol. 5, no. 5, pp. 430-436, 2002.

[6] B. E. Czikkel and D. P. Maxwell, "NtGRAS1, a novel stressinduced member of the GRAS family in tobacco, localizes to the nucleus," Journal of Plant Physiology, vol. 164, no. 9, pp. 1220-1230, 2007.

[7] S. Lee, Y.-M. Woo, S.-I. Ryu et al., "Further characterization of a rice AGL12 group MADS-box gene, OsMADS26," Plant Physiology, vol. 147, no. 1, pp. 156-168, 2008.
[8] X. Li, Q. Qian, Z. Fu et al., "Control of tillering in rice," Nature, vol. 422, no. 6932, pp. 618-621, 2003.

[9] P. Torres-Galea, L. F. Huang, N. H. Chua, and C. Bolle, "The GRAS protein SCL13 is a positive regulator of phytochromedependent red light signaling, but can also modulate phytochrome A responses," Molecular Genetics and Genomics, vol. 276, no. 1, pp. 13-30, 2006.

[10] K. Morohashi, M. Minami, H. Takase, Y. Hotta, and K. Hiratsuka, "Isolation and characterization of a novel GRAS gene that regulates meiosis-associated gene expression," Journal of Biological Chemistry, vol. 278, no. 23, pp. 2086520873, 2003.

[11] M. Gao, I. Parkin, D. Lydiate, and A. Hannoufa, "An auxinresponsive SCARECROW-like transcriptional activator interacts with histone deacetylase," Plant Molecular Biology, vol. 55, no. 3, pp. 417-431, 2004.

[12] C. Sánchez, J. M. Vielba, E. Ferro et al., "Two SCARECROW-LIKE genes are induced in response to exogenous auxin in rooting-competent cuttings of distantly related forest species," Tree Physiology, vol. 27, no. 10, pp. 1459-1470, 2007.

[13] A. L. Silverstone, C. N. Ciampaglio, and T. Sun, "The Arabidopsis RGA gene encodes a transcriptional regulator repressing the gibberellin signal transduction pathway," The Plant Cell, vol. 10, no. 2, pp. 155-169, 1998.

[14] S. Lee, H. Cheng, K. E. King et al., "Gibberellin regulates Arabidopsis seed germination via RGL2,a GAI/RGA-like gene whose expression is up-regulated following imbibition," Genes \& Development, vol. 16, no. 5, pp. 646-658, 2002.

[15] X. Liu and A. Widmer, "Genome-wide comparative analysis of the GRAS gene family in Populus, Arabidopsis and rice," Plant Molecular Biology Reporter, vol. 32, no. 6, pp. 1129-1145, 2014.

[16] C. Tian, P. Wan, S. Sun, J. Li, and M. Chen, "Genome-wide analysis of the GRAS gene family in rice and Arabidopsis," Plant Molecular Biology, vol. 54, no. 4, pp. 519-532, 2004.

[17] C. Bolle, "The role of GRAS proteins in plant signal transduction and development," Planta, vol. 218, no. 5, pp. 683-692, 2004.

[18] L. D. Pysh, J. W. Wysocka-Diller, C. Camilleri, D. Bouchez, and P. N. Benfey, "The GRAS gene family in Arabidopsis: sequence characterization and basic expression analysis of the SCARECROW-LIKE genes," The Plant Journal, vol. 18, no. 1, pp. 111-119, 1999.

[19] X. Sun, W. T. Jones, and E. H. A. Rikkerink, "GRAS proteins: the versatile roles of intrinsically disordered proteins in plant signalling," Biochemical Journal, vol. 442, no. 1, pp. 1-12, 2012.

[20] Y. Yuan, L. Fang, S. K. Karungo et al., "Overexpression of VaPAT1, a GRAS transcription factor from Vitis amurensis, confers abiotic stress tolerance in Arabidopsis," Plant Cell Reports, vol. 35, no. 3, pp. 655-666, 2016.

[21] H. S. Ma, D. Liang, P. Shuai, X. L. Xia, and W. L. Yin, “The salt- and drought-inducible poplar GRAS protein SCL7 confers salt and drought tolerance in Arabidopsis thaliana," Journal of Experimental Botany, vol. 61, no. 14, pp. 40114019, 2010.

[22] M. Mayrose, S. K. Ekengren, S. Melech-Bonfil, G. B. Martin, and G. Sessa, "A novel link between tomato GRAS genes, plant disease resistance and mechanical stress response," Molecular Plant Pathology, vol. 7, no. 6, pp. 593-604, 2006. 
[23] K. Xu, S. Chen, T. Li et al., "OsGRAS23, a rice GRAS transcription factor gene, is involved in drought stress response through regulating expression of stress-responsive genes," BMC Plant Biology, vol. 15, no. 1, 2015.

[24] J. Curaba, M. Talbot, Z. Li, and C. Helliwell, “Over-expression of microRNA171 affects phase transitions and floral meristem determinancy in barley," BMC Plant Biology, vol. 13, no. 1, p. 6, 2013.

[25] T. Fan, X. Li, W. Yang, K. Xia, J. Ouyang, and M. Zhang, "Rice osa-miR $171 \mathrm{c}$ mediates phase change from vegetative to reproductive development and shoot apical meristem maintenance by repressing four OsHAM transcription factors," PLoS One, vol. 10, no. 5, article e0125833, 2015.

[26] X. Y. Xue, B. Zhao, L. M. Chao et al., "Interaction between two timing microRNAs controls trichome distribution in Arabidopsis," PLoS Genetics, vol. 10, no. 4, article e1004266, 2014.

[27] Z. Ma, X. Hu, W. Cai et al., "Arabidopsis miR171-targeted scarecrow-like proteins bind to GT cis-elements and mediate gibberellin-regulated chlorophyll biosynthesis under light conditions," PLoS Genetics, vol. 10, no. 8, p. e1004519, 2014.

[28] E. W. Hwang, S. J. Shin, B. K. Yu, M. O. Byun, and H. B. Kwon, "miR171 family members are involved in drought response in Solanum tuberosum," Journal of Plant Biology, vol. 54, no. 1, pp. 43-48, 2011.

[29] M. Kantar, T. Unver, and H. Budak, "Regulation of barley miRNAs upon dehydration stress correlated with target gene expression," Functional \& Integrative Genomics, vol. 10, no. 4, pp. 493-507, 2010.

[30] Y. Kong, A. A. Elling, B. Chen, and X. Deng, "Differential expression of microRNAs in maize inbred and hybrid lines during salt and drought stress," American Journal of Plant Sciences, vol. 1, no. 2, pp. 69-76, 2010.

[31] H. H. Liu, X. Tian, Y. J. Li, C. A. Wu, and C. C. Zheng, "Microarray-based analysis of stress-regulated microRNAs in Arabidopsis thaliana," RNA, vol. 14, no. 5, pp. 836-843, 2008.

[32] J.-O. Heo, K. S. Chang, I. A. Kim et al., "Funneling of gibberellin signaling by the GRAS transcription regulator SCARECROW-LIKE 3 in the Arabidopsis root," Proceedings of the National Academy of Sciences of the United States of America, vol. 108, no. 5, pp. 2166-2171, 2011.

[33] Z.-L. Zhang, M. Ogawa, C. M. Fleet et al., "SCARECROWLIKE 3 promotes gibberellin signaling by antagonizing master growth repressor DELLA in Arabidopsis," Proceedings of the National Academy of Sciences of the United States of America, vol. 108, no. 5, pp. 2160-2165, 2011.

[34] J. Peng, P. Carol, D. E. Richards et al., "The Arabidopsis GAI gene defines a signaling pathway that negatively regulates gibberellin responses," Genes \& Development, vol. 11, no. 23, pp. 3194-3205, 1997.

[35] E. H. Colebrook, S. G. Thomas, A. L. Phillips, and P. Hedden, "The role of gibberellin signalling in plant responses to abiotic stress," Journal of Experimental Biology, vol. 217, no. 1, pp. 6775, 2014.

[36] P. Achard, J. P. Renou, R. Berthomé, N. P. Harberd, and P. Genschik, "Plant DELLAs restrain growth and promote survival of adversity by reducing the levels of reactive oxygen species," Current Biology, vol. 18, no. 9, pp. 656-660, 2008.

[37] F. Della Rovere, L. Fattorini, S. D'Angeli et al., "Arabidopsis SHR and SCR transcription factors and AUX1 auxin influx carrier control the switch between adventitious rooting and xylogenesis in planta and in in vitro cultured thin cell layers," Annals of Botany, vol. 115, no. 4, pp. 617-628, 2015.

[38] M. Jain and J. P. Khurana, "Transcript profiling reveals diverse roles of auxin-responsive genes during reproductive development and abiotic stress in rice," The FEBS Journal, vol. 276, no. 11, pp. 3148-3162, 2009.

[39] X. Fu and N. P. Harberd, "Auxin promotes Arabidopsis root growth by modulating gibberellin response," Nature, vol. 421, no. 6924, pp. 740-743, 2003.

[40] I. A. Paponov, M. Paponov, W. Teale et al., "Comprehensive transcriptome analysis of auxin responses in Arabidopsis," Molecular Plant, vol. 1, no. 2, pp. 321-337, 2008.

[41] W. Huang, Z. Xian, X. Kang, N. Tang, and Z. Li, "Genomewide identification, phylogeny and expression analysis of GRAS gene family in tomato," BMC Plant Biology, vol. 15, no. 1, p. 209, 2015.

[42] Y. Liu, W. Huang, Z. Xian et al., "Overexpression of SlGRAS40 in tomato enhances tolerance to abiotic stresses and influences auxin and gibberellin signaling," Frontiers in Plant Science, vol. 8, pp. 1-17, 2017.

[43] W. Huang, S. Peng, Z. Xian et al., "Overexpression of a tomato miR171 target gene SlGRAS24 impacts multiple agronomical traits via regulating gibberellin and auxin homeostasis," Plant Biotechnology Journal, vol. 15, no. 4, pp. 472-488, 2017.

[44] J. J. Fillatti, J. Kiser, R. Rose, and L. Comai, "Efficient transfer of a glyphosate tolerance gene into tomato using a binary agrobacterium tumefaciens vector," Bio/Technology, vol. 5, no. 7 , pp. 726-730, 1987.

[45] H. Wang, B. Jones, Z. Li et al., "The tomato Aux / IAA transcription factor IAA9 is involved in fruit development and leaf morphogenesis," Plant Cell, vol. 17, no. 10, pp. 2676-2692, 2005.

[46] Y. Pan, G. B. Seymour, C. Lu, Z. Hu, X. Chen, and G. Chen, "An ethylene response factor (ERF5) promoting adaptation to drought and salt tolerance in tomato," Plant Cell Reports, vol. 31, no. 2, pp. 349-360, 2012.

[47] W. Huang, Z. Xian, G. Hu, and Z. Li, "SlAGO4A, a core factor of RNA-directed DNA methylation (RdDM) pathway, plays an important role under salt and drought stress in tomato," Molecular Breeding, vol. 36, no. 3, pp. 1-13, 2016.

[48] L. Wang, Y. X. Mai, Y. C. Zhang, Q. Luo, and H. Q. Yang, "MicroRNA171c-targeted SCL6-II, SCL6-III, and SCL6-IV genes regulate shoot branching in Arabidopsis," Molecular Plant, vol. 3, no. 5, pp. 794-806, 2010.

[49] S. Zhou, Z. Hu, F. Li et al., "Manipulation of plant architecture and flowering time by down-regulation of the GRAS transcription factor SIGRAS26 in Solanum lycopersicum," Plant Science, vol. 271, pp. 81-93, 2018.

[50] M. Farooq, A. Wahid, D.-J. Lee, O. Ito, and K. H. M. Siddique, "Advances in drought resistance of rice," Critical Reviews in Plant Sciences, vol. 28, no. 4, pp. 199-217, 2009.

[51] M. Seki, M. Narusaka, J. Ishida et al., "Monitoring the expression profiles of 7000 Arabidopsis genes under drought, cold and high-salinity stresses using a full-length cDNA microarray," The Plant Journal, vol. 31, no. 3, pp. 279-292, 2002.

[52] J.-K. Zhu, "Salt and drought stress signal transduction in plants," Annual Review of Plant Biology, vol. 53, no. 1, pp. 247-273, 2002.

[53] C. Lu, Y. Li, A. Chen et al., "LeERF1 improves tolerance to drought stress in tomato (Lycopersicon esculentum) and 
activates downstream stress-responsive genes," African Journal of Biotechnology, vol. 9, pp. 6294-6300, 2010.

[54] X. Cai, C. Zhang, J. Ye et al., "Ectopic expression of FaGalUR leads to ascorbate accumulation with enhanced oxidative stress, cold, and salt tolerance in tomato," Plant Growth Regulation, vol. 76, no. 2, pp. 187-197, 2015.

[55] P. Achard, H. Cheng, L. De Grauwe et al., "Integration of plant responses to environmentally activated phytohormonal signals," Science, vol. 311, no. 5757, pp. 91-94, 2006.

[56] K. Apel and H. Hirt, "REACTIVE OXYGEN SPECIES: metabolism, oxidative stress, and signal transduction," Annual Review of Plant Biology, vol. 55, no. 1, pp. 373-399, 2004.

[57] S.-W. Zhang, C.-H. Li, J. Cao et al., "Altered architecture and enhanced drought tolerance in rice via the down-regulation of indole-3-acetic acid by TLD1/OsGH3.13 activation," Plant Physiology, vol. 151, no. 4, pp. 1889-1901, 2009.

[58] Q. Zhang, J. Li, W. Zhang et al., "The putative auxin efflux carrier OsPIN3t is involved in the drought stress response and drought tolerance," The Plant Journal, vol. 72, no. 5, pp. 805-816, 2012.

[59] J. Tyburski, K. Dunajska, P. Mazurek, B. Piotrowska, and A. Tretyn, "Exogenous auxin regulates $\mathrm{H}_{2} \mathrm{O}_{2}$ metabolism in roots of tomato (Lycopersicon esculentum Mill.) seedlings affecting the expression and activity of CuZn-superoxide dismutase, catalase, and peroxidase," Acta Physiologiae Plantarum, vol. 31, no. 2, pp. 249-260, 2009.

[60] A. Dill, S. G. Thomas, J. Hu, C. M. Steber, and T. P. Sun, “The Arabidopsis F-box protein SLEEPY1 targets gibberellin signaling repressors for gibberellin-induced degradation," The Plant Cell, vol. 16, no. 6, pp. 1392-1405, 2004.

[61] J. Li, W. Sima, B. Ouyang et al., "Tomato SlDREB gene restricts leaf expansion and internode elongation by downregulating key genes for gibberellin biosynthesis," Journal of Experimental Botany, vol. 63, no. 18, pp. 6407-6420, 2012.

[62] S. Huang, A. S. Raman, J. E. Ream, H. Fujiwara, R. E. Cerny, and S. M. Brown, "Overexpression of 20-oxidase confers a gibberellin-overproduction phenotype in Arabidopsis," Plant Physiology, vol. 118, no. 3, pp. 773-781, 1998.

[63] N. García-Hurtado, E. Carrera, O. Ruiz-Rivero et al., “The characterization of transgenic tomato overexpressing gibberellin 20-oxidase reveals induction of parthenocarpic fruit growth, higher yield, and alteration of the gibberellin biosynthetic pathway," Journal of Experimental Botany, vol. 63, no. 16, pp. 5803-5813, 2012.

[64] M. J. Gao, X. Li, J. Huang et al., "SCARECROW-LIKE15 interacts with HISTONE DEACETYLASE19 and is essential for repressing the seed maturation programme," Nature Communications, vol. 6, no. 1, 2015.

[65] E. Benková and J. Hejátko, "Hormone interactions at the root apical meristem," Plant Molecular Biology, vol. 69, no. 4, pp. 383-396, 2009.

[66] S. Perilli, R. Di Mambro, and S. Sabatini, "Growth and development of the root apical meristem," Current Opinion in Plant Biology, vol. 15, no. 1, pp. 17-23, 2012.

[67] E. Shani, O. Yanai, and N. Ori, "The role of hormones in shoot apical meristem function," Current Opinion in Plant Biology, vol. 9, no. 5, pp. 484-489, 2006.

[68] D. Weiss and N. Ori, "Mechanisms of cross talk between gibberellin and other hormones," Plant Physiology, vol. 144, no. 3, pp. 1240-1246, 2007.
[69] E. M. Engstrom, C. M. Andersen, J. Gumulak-Smith et al., "Arabidopsis homologs of the Petunia HAIRY MERISTEM gene are required for maintenance of shoot and root indeterminacy," Plant Physiology, vol. 155, no. 2, pp. 735-750, 2011.

[70] M. Fambrini, L. Mariotti, S. Parlanti, M. Salvini, and C. Pugliesi, "A GRAS-like gene of sunflower (Helianthus annuus L.) alters the gibberellin content and axillary meristem outgrowth in transgenic Arabidopsis plants," Plant Biology, vol. 17 , no. 6 , pp. 1123-1134, 2015. 


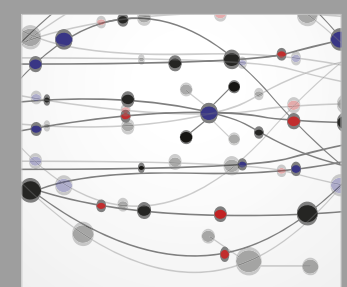

The Scientific World Journal
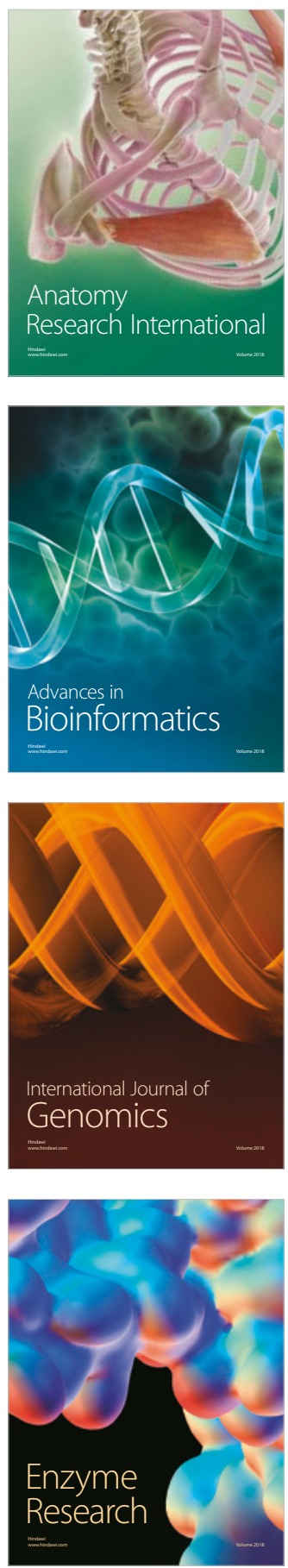
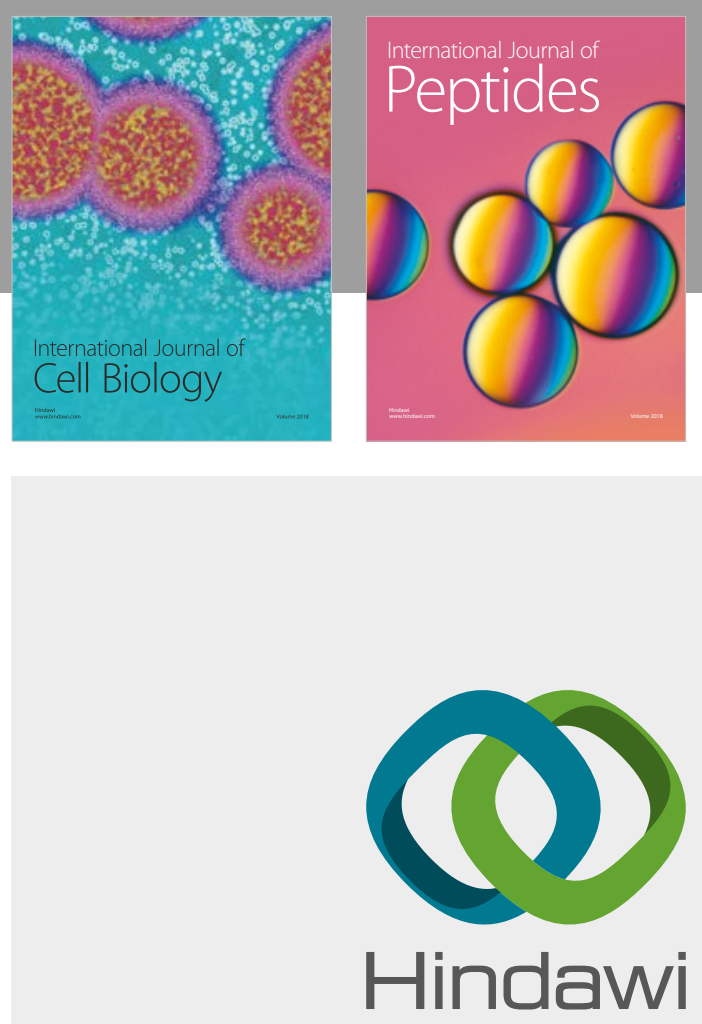

Submit your manuscripts at

www.hindawi.com
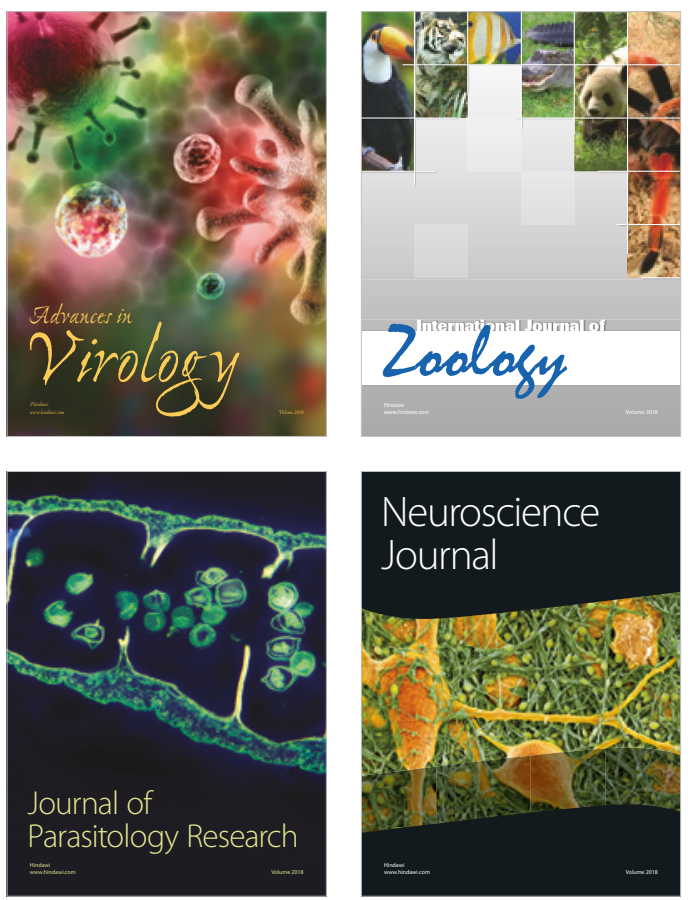
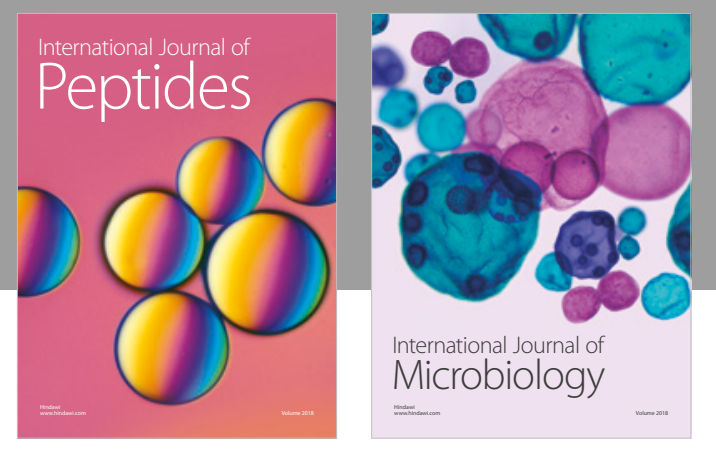

nternational Journal of Microbiology
Journal of
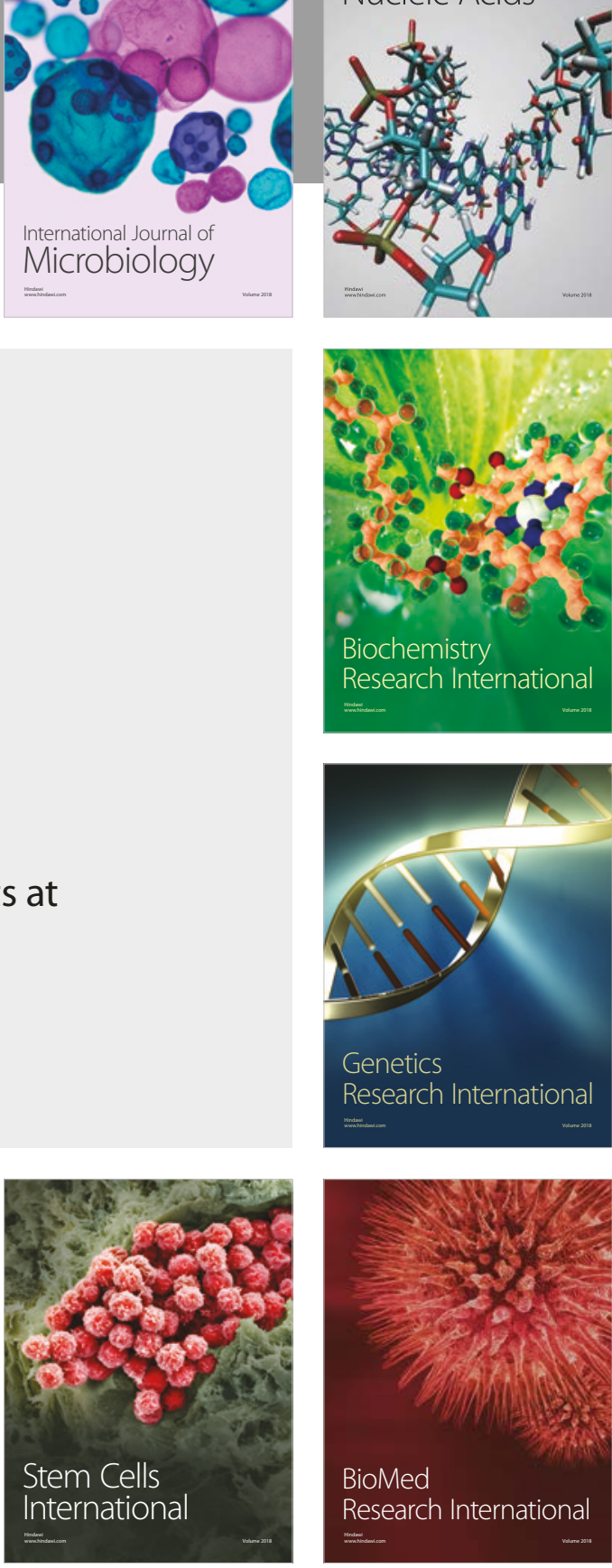
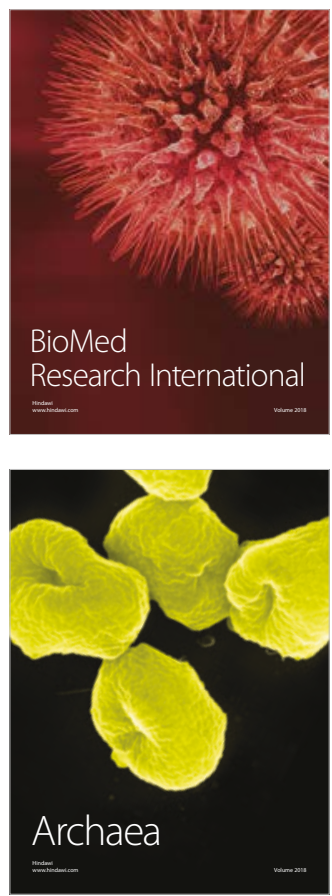